KUNS-1721

hep-th/0106051

\title{
Off-Shell Formulation of Supergravity on Orbifold
}

\author{
Tomoyuki Fujita, 因 Taichiro Kugo甩 and Keisuke OHASH米 \\ Department of Physics, Kyoto University, Kyoto 606-8502
}

\begin{abstract}
An off-shell formulation is given for the "supersymmetry in singular spaces" which has recently been developed in an on-shell formalism by Bergshoeff, Kallosh and Van Proeyen using supersymmetry singlet 'coupling constant' field and 4-form multiplier field in five-dimensional space-time. We present this formulation for a general supergravity-Yang-Mills-hypermultiplet coupled system compactified on an orbifold $S^{1} / Z_{2}$. Relations between the bulk cosmological constant and brane tensions of the boundary planes are discussed.
\end{abstract}

\footnotetext{
*E-mail: fujita@gauge.scphys.kyoto-u.ac.jp

** E-mail: kugo@gauge.scphys.kyoto-u.ac.jp

*** E-mail: keisuke@gauge.scphys.kyoto-u.ac.jp
} 


\section{$\S 1$. Introduction}

Concerning the supersymmetrization of the Randal-Sundrum scenario $\$$ on the orbifold $S^{1} / Z_{2}$, there have appeared two distinct approaches; one is due to Altendorfer, Bagger and Nemeschansky 22, 3) and another is due to Falkowski, Lalak and Pokorsky. 14 The difference resides in the point that the $U(1)_{R}$ gauge coupling constant $g$ and the gravitino 'mass term' change their signs across the branes in the latter approach while they do not in the former approach. (So the brane in the latter approach resembles to a thin limit of the domain wall.) It is only in the latter approach that the supersymmetry requirement can give relations between the cosmological constant in the bulk space and the brane tensions of the two boundary planes. These relations are exactly the same relations which were required for the existence of the Randal-Sundrum's warp solution.11 Moreover it is also the latter case that is expected to appear from the heterotic M-theory on $S^{1} / Z_{2}$ 国 after the reduction to five dimensions by compactifying on a Calabi-Yau 3-fold.

In a paper entitled "Supersymmetry in Singular Spaces", Bergshoeff, Kallosh and Van Proeyen (BKVP) have given an interesting formulation for realizing 'dynamically' this situation of the coupling constant changing its sign across a brane. Namely, they considered the Maxwell/Einstein gauged supergravity system in five dimensions and lifted the gauge coupling constant $g$ of the $U(1)_{R}$ to a supersymmetry singlet field $G(x)$. Then, with introducing a 4-form gauge field $H_{\mu \nu \rho \sigma}$ also, they succeeded in constructing a supersymmetric action for the system on an $S^{1} / Z_{2}$ orbifold and realized the changing sign coupling 'constant' $G(y)=g \epsilon(y)$ as the solution of the equation of motion.

However their construction is heuristic and is presented only in an on-shell formulation for the pure Maxwell/Einstein gauged supergravity system. It is thus unclear how it becomes changed when the system is varied. The purpose of this paper is, therefore, to give an offshell generalization for the BKVP formulation. In our formulation, the coupling field $G(x)$ appears as a ratio of the scalar component fields of two vector multiplets, and the 4-form gauge field $H_{\mu \nu \rho \sigma}$ is supplied essentially as a scalar component field of a linear multiplet.

This paper is organized as follows. In $\S 2$ we briefly explain the form of invariant offshell action for the general supergravity-Yang-Mills-hypermultiplet system in 5D, 9) which we consider in this paper. Next in $\S 3$, we present a new form of linear multiplet in which the constrained vector and auxiliary scalar components are rewritten in terms of 3 -form and 4-form gauge fields, respectively. This was briefly pointed out in Ref. 10) but we present the details here for the first time. Using this new form of linear multiplet as a supermultiplet containing BKVP's 4-form gauge field $H_{\mu \nu \rho \sigma}$, we give an off-shell version of the 4-form gauge field in $5 \mathrm{D}$ bulk. In $\S 4$ we discuss the compactification of the system on orbifold $S^{1} / Z_{2}$ and 
construct a brane action which again gives an off-shell generalization of BKVP's. In $\S 5$, we discuss the relation between the cosmological constant and the brane tensions of the boundary planes, based on the obtained action. We show that various results obtained by previous authors are reproduced from our general results by reducing to simpler systems. Final section 6 is devoted to discussions. Some technical points are treated in Appendix concerning the parametrizations of the target manifold $U(2, q) / U(2) \times U(q)$ of the hypermultiplet scalar fields and the non-linear Lagrangian.

\section{$\S 2$. Supergravity action in five-dimensional bulk}

The invariant action for a general system of Yang-Mills and hypermultiplet matters coupled to supergravity in off-shell formulation was first obtained in Ref. 9), which we refer to as I henceforth, based on the super Poincaré tensor calculus given in Ref. 11). However, the calculation was very tedious there since there was no conformal $S$-supersymmetry. Weyl multiplets in 5D conformal supergravity were constructed very recently by Bergshoeff et al (2) and the full superconformal tensor calculus was presented by Fujita and Ohashi in Ref. 10), which we refer to as II, where it was explained how easily the result of I can be rederived based on the superconformal tensor calculus. We here, therefore, follow the technique developed in II.

We here consider the system of $n+1$ vector multiplets $\boldsymbol{V}^{I}(I=0,1,2, \cdots, n)$ of some gauge group $G$ and $r$ hypermultiplets $\boldsymbol{H}^{\alpha}(\alpha=1,2, \cdots, 2 r)$ which give a certain representation of $G$ with representation matrix $\left(g t_{I}\right)^{\alpha}{ }_{\beta}$. The field contents of the Weyl multiplet, vector multiplet and hypermultiplet are listed in Table. $\mathbb{E}$.

In the superconformal framework, the action obtained in I results if we fix the extraneous gauge freedoms of dilatation $\boldsymbol{D}$, conformal supersymmetry $\boldsymbol{S}$ and special conformal-boost $\boldsymbol{K}$ symmetries by the conditions 10

$$
\boldsymbol{D}: \mathcal{N}=1, \quad \boldsymbol{S}: \Omega^{I i} \mathcal{N}_{I}=0, \quad \boldsymbol{K}: \hat{\mathcal{D}}_{a} \mathcal{N}=0
$$

where $\mathcal{N}(M)$ is the homogeneous cubic function of the scalar component fields $M^{I}$ of the vector multiplets $\boldsymbol{V}^{I}$ which uniquely characterizes the vector part action of the system. We use notations like $\mathcal{N}_{I} \equiv \partial \mathcal{N} / \partial M^{I}, \mathcal{N}_{I J} \equiv \partial \mathcal{N} / \partial M^{I} \partial M^{J}$, etc, and $\hat{\mathcal{D}}_{\mu}$ denotes full superconformal covariant derivative. The $\boldsymbol{Q}$ supersymmetry transformation which preserves these gauge conditions are given by the combination of $\boldsymbol{Q}, \boldsymbol{S}$ and $\boldsymbol{K}$ :

$$
\begin{aligned}
\tilde{\delta}_{Q}(\varepsilon) & =\delta_{Q}(\varepsilon)+\delta_{S}\left(\eta^{i}(\varepsilon)\right)+\delta_{K}\left(\xi_{K}^{a}(\varepsilon)\right), \\
\eta^{i}(\varepsilon) & =-\frac{\mathcal{N}_{I}}{12 \mathcal{N}} \gamma \cdot \hat{F}^{I}(W) \varepsilon^{i}+\frac{\mathcal{N}_{I}}{3 \mathcal{N}} Y^{I i}{ }_{j} \varepsilon^{j}+\frac{\mathcal{N}_{I J}}{3 \mathcal{N}} \Omega^{I i}\left(2 i \bar{\varepsilon} \Omega^{J}\right),
\end{aligned}
$$


Table I. Field contents of the multiplets

\begin{tabular}{ccccc}
\hline \hline field & type & restrictions & $S U(2)$ & Weyl-weight \\
\hline \multicolumn{5}{c}{ Weyl multiplet } \\
\hline$e_{\mu}{ }^{a}$ & boson & fünfbein & $\mathbf{1}$ & -1 \\
$\psi_{\mu}^{i}$ & fermion & $S U(2)$-Majorana & $\mathbf{2}$ & $-\frac{1}{2}$ \\
$b_{\mu}$ & boson & real & $\mathbf{1}$ & 0 \\
$V_{\mu}^{i j}$ & boson & $V_{\mu}^{i j}=V_{\mu}^{j i}=\left(V_{\mu i j}\right)^{*}$ & $\mathbf{3}$ & 0 \\
$v_{a b}$ & boson & real, antisymmetric & $\mathbf{1}$ & 1 \\
$\chi^{i}$ & fermion & $S U(2)$-Majorana & $\mathbf{2}$ & $\frac{3}{2}$ \\
$D^{i}$ & boson & real & $\mathbf{1}$ & 2 \\
\hline \multicolumn{5}{c}{ Vector multiplet $\boldsymbol{V}^{I}$} \\
\hline$W_{\mu}^{I}$ & boson & real gauge field & $\mathbf{1}$ & 0 \\
$M^{I}$ & boson & real scalar & $\mathbf{1}$ & 1 \\
$\Omega^{I i}$ & fermion & $S U(2)$-Majorana & $\mathbf{2}$ & $\frac{3}{2}$ \\
$Y_{i j}^{I}$ & boson & $Y^{I i j}=Y^{I j i}=\left(Y_{i j}^{I}\right)^{*}$ & $\mathbf{3}$ & 2 \\
\hline \multicolumn{5}{c}{$H_{y p e r m u l t i p l e t} \boldsymbol{H}^{\alpha}$} \\
\hline $\mathcal{A}_{i}^{\alpha}$ & boson & $\mathcal{A}_{\alpha}^{i}=\varepsilon^{i j} \mathcal{A}_{j}^{\beta} \rho_{\beta \alpha}=-\left(\mathcal{A}_{i}^{\alpha}\right)^{*}$ & $\mathbf{2}$ & $\frac{3}{2}$ \\
$\zeta^{\alpha}$ & fermion & $\bar{\zeta}^{\alpha} \equiv\left(\zeta_{\alpha}\right)^{\dagger} \gamma_{0}=\zeta^{\alpha \mathrm{T}} C$ & $\mathbf{1}$ & 2 \\
$\mathcal{F}_{i}^{\alpha}$ & boson & $\mathcal{F}_{\alpha}^{i}=-\left(\mathcal{F}_{i}^{\alpha}\right)^{*}$ & $\mathbf{2}$ & $\frac{5}{2}$ \\
\hline \multicolumn{7}{c}{}
\end{tabular}

omitting the expression of unimportant parameter $\xi_{K}^{a}(\varepsilon)$. The resultant $Q$ transformation laws of the Weyl multiplet, the vector multiplet and the hypermultiplet are completely the same as (I 6.8), (I 6.9) and (I 6.10), respectively, given in I, provided that the following translation rules are used (the LHS is the present notations same as in II and the RHS is those in I)

$$
\begin{aligned}
& V_{\mu}^{i j} \leftrightarrow \tilde{V}_{\mu}^{i j}, \quad v_{a b} \leftrightarrow \tilde{v}_{a b}, \quad \frac{\mathcal{N}_{I}}{3 \mathcal{N}} Y^{I i j} \leftrightarrow-\tilde{t}^{i j}, \\
& \chi^{i} \leftrightarrow 16 \tilde{\chi}^{i}+3 \gamma \cdot \hat{\mathcal{R}}^{i}(Q), \quad D \leftrightarrow 8 \tilde{C}-\frac{3}{2} \hat{\mathcal{R}}(M)+2 v^{2}, \\
& \Omega \leftrightarrow \lambda, \quad \zeta_{\alpha} \leftrightarrow \xi_{\alpha}, \quad Y^{I i j} \leftrightarrow \tilde{Y}^{I i j}-M^{I} \tilde{t}^{i j} .
\end{aligned}
$$

We therefore omit those $\boldsymbol{Q}$ transformation rules here, but cite the explicit form of the action for the present supergravity-Yang-Mills-hypermultiplet system:

$$
\begin{aligned}
& \mathcal{L}_{0}=\mathcal{L}_{\text {hyper }}+\mathcal{L}_{\text {vector }}+\mathcal{L}_{\mathrm{C}-\mathrm{S}}+\mathcal{L}_{\text {aux }} \\
& e^{-1} \mathcal{L}_{\text {hyper }}=\nabla^{a} \mathcal{A}_{i}^{\bar{\alpha}} \nabla_{a} \mathcal{A}_{\alpha}^{i}-2 i \bar{\zeta}^{\bar{\alpha}}(\not \nabla+g M) \zeta_{\alpha}
\end{aligned}
$$




$$
\begin{aligned}
& +\mathcal{A}_{i}^{\bar{\alpha}}(g M)^{2}{ }_{\alpha}{ }^{\beta} \mathcal{A}_{\beta}^{i}-4 i \bar{\psi}_{a}^{i} \gamma^{b} \gamma^{a} \zeta_{\alpha} \nabla_{b} \mathcal{A}_{i}^{\bar{\alpha}}-2 i \bar{\psi}_{a}^{(i} \gamma^{a b c} \psi_{c}^{j)} \mathcal{A}_{j}^{\bar{\alpha}} \nabla_{b} \mathcal{A}_{\alpha i} \\
& +\mathcal{A}_{i}^{\bar{\alpha}}\left(8 i g \bar{\Omega}_{\alpha \beta}^{i} \zeta^{\beta}-4 i g \bar{\psi}_{a}^{i} \gamma^{a} M_{\alpha \beta} \zeta^{\beta}\right. \\
& \left.+4 i g \bar{\psi}_{a}^{(i} \gamma^{a} \Omega_{\alpha \beta}^{j)} \mathcal{A}_{j}^{\beta}-2 i g \bar{\psi}_{a}^{(i} \gamma^{a b} \psi_{b}^{j)} M_{\alpha \beta} \mathcal{A}_{j}^{\beta}\right) \\
& +\bar{\psi}_{a} \gamma_{b} \psi_{c} \bar{\zeta}^{\bar{\alpha}} \gamma^{a b c} \zeta_{\alpha}-\frac{1}{2} \bar{\psi}^{a} \gamma^{b c} \psi_{a} \bar{\zeta}^{\bar{\alpha}} \gamma_{b c} \zeta_{\alpha} \\
& e^{-1} \mathcal{L}_{\text {vector }}=-\frac{1}{2} R(\omega)-2 i \bar{\psi}_{\mu} \gamma^{\mu \nu \rho} \nabla_{\nu} \psi_{\rho}+\left(\bar{\psi}_{a} \psi_{b}\right)\left(\bar{\psi}_{c} \gamma^{a b c d} \psi_{d}+\bar{\psi}^{a} \psi^{b}\right) \\
& -\mathcal{N}_{I}\left(i g[\bar{\Omega}, \Omega]^{I}-\frac{i}{4} \bar{\psi}_{c} \gamma^{a b c d} \psi_{d} F_{a b}(W)^{I}\right) \\
& +a_{I J}\left(\begin{array}{c}
-\frac{1}{4} F(W)^{I} \cdot F(W)^{J}+\frac{1}{2} \nabla_{a} M^{I} \nabla^{a} M^{J} \\
+2 i \bar{\Omega}^{I} \not \nabla \Omega^{J}+i \bar{\psi}_{a}(\gamma \cdot F(W)-2 \not \nabla M)^{I} \gamma^{a} \Omega^{J} \\
-2\left(\bar{\Omega}^{I} \gamma^{a} \gamma^{b c} \psi_{a}\right)\left(\bar{\psi}_{b} \gamma_{c} \Omega^{J}\right)+2\left(\bar{\Omega}^{I} \gamma^{a} \gamma^{b} \psi_{a}\right)\left(\bar{\psi}_{b} \Omega^{J}\right)
\end{array}\right) \\
& -\mathcal{N}_{I J K}\left(\begin{array}{c}
-i \bar{\Omega}^{I} \frac{1}{4} \gamma \cdot F(W)^{J} \Omega^{K} \\
+\frac{2}{3}\left(\bar{\Omega}^{I} \gamma^{a b} \Omega^{J}\right)\left(\bar{\psi}_{a} \gamma_{b} \Omega^{K}\right)+\frac{2}{3}\left(\bar{\psi}^{i} \cdot \gamma \Omega^{I j}\right)\left(\bar{\Omega}_{(i}^{J} \Omega_{j)}^{K}\right)
\end{array}\right) \\
& +\frac{1}{8}\left(2 \bar{\psi}_{a} \psi_{b}+\bar{\zeta}^{\bar{\alpha}} \gamma_{a b} \zeta_{\alpha}+a_{I J} \bar{\Omega}^{I} \gamma_{a b} \Omega^{J}\right)^{2} \\
& +i \frac{1}{4} \mathcal{N}_{I} F(W)^{I}\left(2 \bar{\psi}_{a} \psi_{b}+\bar{\zeta}^{\bar{\alpha}} \gamma_{a b} \zeta_{\alpha}+a_{I J} \bar{\Omega}^{I} \gamma_{a b} \Omega^{J}\right) \\
& +\left(\mathcal{A}^{\bar{\alpha} i} \nabla_{a} \mathcal{A}_{\alpha}^{j}+i a_{I J} \bar{\Omega}^{I i} \gamma_{a} \Omega^{J j}\right)^{2} \\
& \mathcal{L}_{\mathrm{C}-\mathrm{S}}=\frac{1}{8} c_{I J K} \epsilon^{\lambda \mu \nu \rho \sigma} W_{\lambda}^{I}\left(F_{\mu \nu}^{J}(W) F_{\rho \sigma}^{K}(W)+\frac{1}{2} g\left[W_{\mu}, W_{\nu}\right]^{J} F_{\rho \sigma}^{K}(W)\right. \\
& \left.+\frac{1}{10} g^{2}\left[W_{\mu}, W_{\nu}\right]^{J}\left[W_{\rho}, W_{\sigma}\right]^{K}\right) .
\end{aligned}
$$

Here $\nabla_{\mu}$ denotes the derivative covariant only with respect to local-Lorentz and group transformations, and the metric $a_{I J}$ in the vector multiplet kinetic term is

$$
a_{I J} \equiv-\frac{1}{2} \frac{\partial^{2}}{\partial M^{I} \partial M^{J}} \ln \mathcal{N}=-\frac{1}{2 \mathcal{N}}\left(\mathcal{N}_{I J}-\frac{\mathcal{N}_{I} \mathcal{N}_{J}}{\mathcal{N}}\right)
$$

The barred group index $\bar{\alpha}$ of the hypermultiplet is defined to be $\mathcal{A}^{\bar{\alpha}} \equiv \mathcal{A}^{\beta} d_{\beta}{ }^{\alpha}$ by using the hypermultiplet metric matrix $d_{\alpha}{ }^{\beta}$ which is given in the standard form 13 as

$$
d_{\alpha}{ }^{\beta}=\left(\begin{array}{cc}
\mathbf{1}_{2 p} & \\
& -\mathbf{1}_{2 q}
\end{array}\right) \quad p+q=r .
$$

Since this implies $\mathcal{A}_{i}^{\bar{\alpha}} \mathcal{A}_{\alpha}^{i}=-\sum_{\alpha=1}^{2 p}\left|\mathcal{A}_{i}^{\alpha}\right|^{2}+\sum_{\alpha=2 p+1}^{2(p+q)}\left|\mathcal{A}_{i}^{\alpha}\right|^{2}$, the first $2 p$ components of the hypermultiplet carry negative metric and are called compensator which will be eliminated eventually by the gauge-fixing of suitable gauge symmetries. We consider only $p=1$ and $p=2$ cases explicitly in this paper.

The last part of the lagrangian, $\mathcal{L}_{\text {aux }}$, is the terms of the auxiliary fields which are written almost in the perfect square forms and vanish on shell, aside from the $Y_{i j}^{I}$-terms to which 
additional contributions will appear later:

$$
\begin{aligned}
e^{-1} \mathcal{L}_{\mathrm{aux}}= & D^{\prime}\left(\mathcal{A}^{2}+2\right)-8 i \bar{\chi}^{\prime i} \mathcal{A}_{i}^{\bar{\alpha}} \zeta_{\alpha}+\left(Y_{i j}^{I} \text {-terms }\right) \\
& +2\left(v-v_{\mathrm{sol}}\right)^{a b}\left(v-v_{\mathrm{sol}}\right)_{a b}-\left(V_{\mu}-V_{\mu \mathrm{sol}}\right)^{i j}\left(V^{\mu}-V_{\mathrm{sol}}^{\mu}\right)_{i j} \\
& +\left(1-A^{2} / \alpha^{2}\right)\left(\mathcal{F}_{i}^{\bar{\alpha}}-\mathcal{F}_{\mathrm{sol} i}^{\bar{\alpha}}\right)\left(\mathcal{F}_{\alpha}^{i}-\mathcal{F}_{\mathrm{sol} \alpha}^{i}\right), \\
\left(Y_{i j}^{I} \text {-terms }\right) & =-\frac{1}{2} \mathcal{N}_{I J} Y_{i j}^{I} Y^{J i j}+Y_{i j}^{I} \mathcal{Y}_{I}^{i j},
\end{aligned}
$$

where

$$
\begin{aligned}
\mathcal{Y}_{I}^{i j} & =2 \mathcal{A}_{\alpha}^{(i}\left(g t_{I}\right)^{\bar{\alpha} \beta} \mathcal{A}_{\beta}^{j)}+i \mathcal{N}_{I J K} \bar{\Omega}^{J i} \Omega^{K j} \\
D^{\prime} & \equiv \frac{1}{8} D+\frac{3}{16} \hat{R}(M)-\frac{1}{4} v^{2}-\frac{i}{8} \bar{\psi} \cdot \gamma \chi+\frac{3 i}{8} \bar{\psi} \cdot \gamma \gamma \cdot \hat{R}(Q)+i \bar{\psi}_{a} \gamma^{a b} \boldsymbol{\Gamma} \psi_{b}, \\
\chi^{\prime i} & \equiv \frac{1}{16} \chi^{i}-\frac{3}{16} \gamma \cdot \hat{R}^{i}(Q)-\frac{1}{2} \gamma^{a} \boldsymbol{\Gamma} \psi_{a}^{i} \\
\boldsymbol{\Gamma} \varepsilon^{i} & \equiv-3 \eta^{i}(\varepsilon)-\gamma^{a} V_{a}{ }_{j} \varepsilon^{j}+\gamma \cdot v \varepsilon^{i}
\end{aligned}
$$

with $\eta^{i}(\varepsilon)$ defined in Eq. $(2 \cdot 2)$. We have omitted the expressions for $V_{\text {sol } a}^{i j}, v_{\text {sol } a b}$ and $\mathcal{F}_{\text {sol } i}^{\alpha}$ which are the same as given in I and we will not need below.

\section{$\S 3$. The invariant action for the four-form gauge field in 5D bulk}

A linear multiplet $\boldsymbol{L}$ consists of an $S U(2)$ triplet boson $L^{i j}$, an $S U(2)$-Majorana spinor $\varphi^{i}$, a constrained vector $E^{a}$ and a real auxiliary scalar $N$. An invariant action formula exists for a pair of an Abelian vector multiplet $\boldsymbol{V}=\left(M, W_{\mu}, \Omega^{i}, Y^{i j}\right)$ and a linear multiplet $\boldsymbol{L}=\left(L^{i j}, \varphi^{i}, E^{a}, N\right)$ which is neutral or charged under the Abelian group of the vector multiplet $\boldsymbol{V}: 10,110$

$$
\begin{aligned}
e^{-1} \mathcal{L}_{\mathrm{VL}}(\boldsymbol{V}, \boldsymbol{L})= & Y^{i j} L_{i j}+2 i \bar{\Omega} \varphi+2 i \bar{\psi}_{i}^{a} \gamma_{a} \Omega_{j} L^{i j} \\
& -\frac{1}{2} W_{a}\left(E^{a}-2 i \bar{\psi}_{b} \gamma^{b a} \varphi+2 i \bar{\psi}_{b}^{(i} \gamma^{a b c} \psi_{c}^{j)} L_{i j}\right) \\
& +\frac{1}{2} M\left(N-2 i \bar{\psi}_{b} \gamma^{b} \varphi-2 i \bar{\psi}_{a}^{(i} \gamma^{a b} \psi_{b}^{j)} L_{i j}\right) .
\end{aligned}
$$

Now we consider the cases where the linear multiplet $\boldsymbol{L}$ is neutral. It was observed in II that this lagrangian density can be consistently written as the following total derivative form of a 4 -form field $H_{\mu \nu \rho \sigma}$ : 因

$$
2 \mathcal{L}_{\mathrm{VL}}(\boldsymbol{V}, \boldsymbol{L})=-\frac{1}{4 !} \epsilon^{\lambda \mu \nu \rho \sigma} \partial_{\lambda}\left(H_{\mu \nu \rho \sigma}-4 W_{\mu} E_{\nu \rho \sigma}\right),
$$

* Note, however, that we are not claiming that the action $\int d^{5} x \mathcal{L}_{\mathrm{VL}}(\boldsymbol{V}, \boldsymbol{L})$ always vanishes. If the action $\int d^{5} x \mathcal{L}_{\mathrm{VL}}(\boldsymbol{V}, \boldsymbol{L})$ is nonzero, then it merely implies that the 4 -form field $H_{\mu \nu \rho \sigma}$ does not vanish at infinity so that the surface term remains finite. 
where $E_{\mu \nu \rho}$ is an unconstrained 3-form field with which the 'divergenceless' constraint on $E^{a}$ is solved in the form 11 .

$$
\begin{aligned}
E^{a} & =\frac{1}{4 !} \epsilon^{a b c d e} \hat{F}_{b c d e}(E), \\
\hat{F}_{\mu \nu \rho \sigma}(E) & =4 \partial_{[\mu} E_{\nu \rho \sigma]}+8 i \bar{\psi}_{[\mu} \gamma_{\nu \rho \sigma]} \varphi+24 i \bar{\psi}_{[\mu}^{i} \gamma_{\nu \rho} \psi_{\sigma]}^{j} L_{i j} .
\end{aligned}
$$

The Eq. (3.2) can be equivalently rewritten as

$$
M N+2 Y_{i j} L^{i j}+4 i \bar{\Omega} \varphi=-\frac{1}{5 !} \epsilon^{a b c d e} \hat{F}_{a b c d e}(H)
$$

using the covariant field strength $\hat{F}_{a b c d e}(H)$ of the 4-form field $H_{\mu \nu \rho \sigma}$ :

$$
\begin{aligned}
\hat{F}_{\lambda \mu \nu \rho \sigma}(H)= & 5 \partial_{[\lambda} H_{\mu \nu \rho \sigma]}-10 F_{[\lambda \mu}(W) E_{\nu \rho \sigma]}-10 i \bar{\psi}_{[\lambda} \gamma_{\mu \nu \rho \sigma]} M \varphi \\
& +20 i \bar{\psi}_{[\lambda}^{i} \gamma_{\mu \nu \rho \sigma]} \lambda^{j} L_{i j}-40 i \bar{\psi}_{[\lambda}^{i} \gamma_{\mu \nu \rho} \psi_{\sigma]}^{j} M L_{i j} .
\end{aligned}
$$

Note the parallelism between Eqs. (3.3) and (3.5). Since the 4-form gauge field $H_{\mu \nu \rho \sigma}$ has $\left(\begin{array}{l}4 \\ 4\end{array}\right)=1$ degree of freedom in $5 \mathrm{D}$, it can be regarded as a replacement of the scalar component $N$ of the linear multiplet $\boldsymbol{L}$, just as the 3 -form gauge field $E_{\mu \nu \rho}$ (possessing $\left(\begin{array}{l}4 \\ 3\end{array}\right)=4$ degrees of freedom in $5 \mathrm{D})$ is the replacement of the constrained vector component $E^{a}$ of $\boldsymbol{L}$. Therefore the linear multiplet $\boldsymbol{L}=\left(L^{i j}, \varphi^{i}, E^{a}, N\right)$ can now be expressed as $\boldsymbol{L}=\left(L^{i j}, \varphi^{i}, E_{\mu \nu \rho}, H_{\mu \nu \rho \sigma}\right)$ by using the 3 -form and 4-form gauge fields. The transformation $\delta \equiv \delta_{Q}(\varepsilon)+\delta_{S}(\eta)+\delta_{E}\left(\Lambda_{\mu \nu}\right)+$ $\delta_{H}\left(\Lambda_{\mu \nu \lambda}\right)$ of these gauge fields are given by

$$
\begin{aligned}
\delta E_{\mu \nu \lambda}= & 3 \partial_{[\mu} \Lambda_{\nu \lambda]}-2 i \bar{\varepsilon} \gamma_{\mu \nu \lambda} \varphi-12 i \bar{\varepsilon}^{i} \gamma_{[\mu \nu} \psi_{\lambda]}^{j} L_{i j} \\
\delta H_{\mu \nu \rho \sigma}= & 4 \partial_{[\mu} \Lambda_{\nu \rho \sigma]}+6 F_{[\mu \nu}(W) \Lambda_{\rho \sigma]}+2 i \bar{\varepsilon} \gamma_{\mu \nu \rho \sigma} M \varphi \\
& -4 i \bar{\varepsilon}^{i} \gamma_{\mu \nu \rho \sigma} \Omega^{j} L_{i j}+16 i \bar{\varepsilon}^{i} \gamma_{[\mu \nu \rho} \psi_{\sigma]}^{j} M L_{i j}+4\left(\delta_{Q}(\varepsilon) W_{[\mu}\right) E_{\nu \rho \sigma]} .
\end{aligned}
$$

It should, however, be kept in mind that this rewriting of the component field $N$ in terms of the 4-form gauge field $H_{\mu \nu \rho \sigma}$ is performed on the 'background' not only of the Weyl multiplet but also of the vector multiplet $\boldsymbol{V}$; that is, the $H_{\mu \nu \rho \sigma}$ component of $\boldsymbol{L}$ depends on $\boldsymbol{V}$, and we use the notation $\left.H_{\mu \nu \rho \sigma}\right|_{\boldsymbol{V}}$ and

$$
\left.\boldsymbol{L}\right|_{\boldsymbol{V}}=\left(L^{i j}, \varphi^{i}, E_{\mu \nu \rho},\left.H_{\mu \nu \rho \sigma}\right|_{\boldsymbol{V}}\right)
$$

to show explicitly the vector multiplet $\boldsymbol{V}$ used in this rewriting.

If we apply the invariant action formula $\mathcal{L}_{\mathrm{VL}}(\boldsymbol{V}, \boldsymbol{L})$ in Eq. (3.1) using the same vector multiplet $\boldsymbol{V}$ as that used in the rewriting $\left.N \rightarrow H_{\mu \nu \rho \sigma}\right|_{\boldsymbol{V}}$ of the linear multiplet $\boldsymbol{L}$, the action takes the total derivative form (3.2). However, we can use different vector multiplets, $\boldsymbol{V}_{S}$ 
and $\boldsymbol{V}_{R}$, for the former and the latter, respectively. Then the invariant action formula $(3 \cdot 1)$ gives the desired 4 -form field action:

$$
\begin{aligned}
\mathcal{L}_{4 \text {-form }}= & \mathcal{L}_{\mathrm{VL}}\left(\boldsymbol{V}_{S},\left.\boldsymbol{L}\right|_{\boldsymbol{V}_{R}}\right) \\
= & e\left(\left(Y_{S}^{i j}-G Y_{R}^{i j}\right) L_{i j}+2 i\left(\bar{\Omega}_{S}^{i}-G \bar{\Omega}_{R}^{i}\right) \varphi_{i}+2 i \bar{\psi}_{i}^{a} \gamma_{a}\left(\Omega_{S j}-G \Omega_{R j}\right) L^{i j}\right) \\
& -\frac{1}{4 !} \epsilon^{\lambda \mu \nu \rho \sigma}\left\{\left(F_{\lambda \mu}\left(W_{S}\right)-G F_{\lambda \mu}\left(W_{R}\right)\right) E_{\nu \rho \sigma}+\frac{1}{2} G \partial_{\lambda} H_{\mu \nu \rho \sigma}\right\},
\end{aligned}
$$

where $G \equiv M_{S} / M_{R}$. This form is most easily obtained by rewriting as

$$
\mathcal{L}_{\mathrm{VL}}\left(\boldsymbol{V}_{S},\left.\boldsymbol{L}\right|_{\boldsymbol{V}_{R}}\right)=\mathcal{L}_{\mathrm{VL}}\left(\boldsymbol{V}_{S}-G \boldsymbol{V}_{R},\left.\boldsymbol{L}\right|_{V_{R}}\right)+G \mathcal{L}_{\mathrm{VL}}\left(\boldsymbol{V}_{R},\left.\boldsymbol{L}\right|_{\boldsymbol{V}_{R}}\right)
$$

Then, the first term can be calculated by the formula (3.1) plaguing $\boldsymbol{V}_{S}-G \boldsymbol{V}_{R}=\left(0, W_{S \mu}-\right.$ $\left.G W_{R \mu}, \Omega_{S}^{i}-G \Omega_{R}^{i}, Y_{S}^{i j}-G Y_{R}^{i j}\right)$ and using Eq. (3·3), and the second term gives $G$ times the total derivative form $(3 \cdot 2)$.

This Eq. (3.9) gives the desired 4-form field action, which gives a off-shell generalization of the corresponding one by BKVP. W With this action, all the component fields of the linear multiplet $\left.\boldsymbol{L}\right|_{V_{R}}$ now play the role of Lagrange multipliers; in particular, the variation of the 4-form field $H_{\mu \nu \rho \sigma}$ constrains the field ratio $G(x) \equiv M_{S}(x) / M_{R}(x)$ to be a constant which plays the role of a coupling constant $g_{R}$ :

$$
\partial_{\mu} G(x)=0 \rightarrow G(x)=g_{R}(\text { constant })
$$

The other equations given by the variation of other components read

$$
Y_{S}^{i j}=G Y_{R}^{i j}, \quad \Omega_{S j}=G \Omega_{R j}, \quad F_{\lambda \mu}\left(W_{S}\right)=G F_{\lambda \mu}\left(W_{R}\right)
$$

It would be appropriate to explain here how the gauged $U(1)_{R}$ supergravity is constructed with these two vector multiplets $\boldsymbol{V}_{S}$ and $\boldsymbol{V}_{R}$ in the present formulation. We identify the vector multiplet $\boldsymbol{V}_{R}$ with the $U(1)_{R}$ gauge multiplet which is generally given by a linear combination of the (Abelian) vector multiplets $\boldsymbol{V}^{I}$ :

$$
\boldsymbol{V}_{R} \equiv V_{I} \boldsymbol{V}^{I}, \quad \text { i.e. } \quad\left(M_{R}, W_{R \mu}, \cdots\right)=\left(V_{I} M^{I}, V_{I} W_{\mu}^{I}, \cdots\right)
$$

We use the index $I$ to denote all the vector multiplets other than $\boldsymbol{V}_{S}$. In the paper I in which no $\boldsymbol{V}_{S}$ appeared, the $U(1)_{R}$ gauge multiplet $\boldsymbol{V}_{R} \equiv V_{I} \boldsymbol{V}^{I}$ was made couple only to the hypermultiplet compensator in the form

$$
\mathcal{D}_{\mu} \mathcal{A}_{i}^{a}=\partial_{\mu} \mathcal{A}_{i}^{a}-V_{\mu i j} \mathcal{A}^{a j}-g_{R} W_{R \mu}(i \vec{q} \cdot \vec{\sigma})^{a}{ }_{b} \mathcal{A}_{i}^{b}
$$


where the indices $a, b$ denote 1 and 2 , the first two values of $\alpha=1,2, \cdots$ corresponding to the first compensator and $\vec{q}$ is any constant isovector of unit length, $\left|\vec{q}^{2}\right|=1$. Then, after the $S U(2)$ gauge of the index $i$ is fixed by the condition $\mathcal{A}_{i}^{a} \propto \delta_{i}^{a}$, the kinetic term $-\left|\mathcal{D}_{\mu} \mathcal{A}_{i}^{a}\right|^{2}$ of the compensator $\mathcal{A}_{i}^{a}$ gives a square term in $V_{\mu}^{\mathrm{N} i j}=V_{\mu}^{i j}-g_{R} W_{R \mu}(i \vec{q} \cdot \vec{\sigma})^{i j}$ so that the auxiliary $S U(2)$ gauge field $V_{\mu}^{i j}$ contained in the covariant derivative $\mathcal{D}_{\mu}$ in all the other places become replaced by $V_{\mu}^{\mathrm{N} i j}+g_{R} W_{R \mu}(i \vec{q} \cdot \vec{\sigma})^{i j}$, thus yielding a universal coupling of $W_{R \mu}$ to the $U(1)_{R}$ subgroup of $S U(2)$ with index $i$. (See I for more details.)

Here, on the other hand, $\boldsymbol{V}_{S}$ eventually becomes the $U(1)_{R}$ gauge multiplet $\boldsymbol{V}_{R}$ times the 'coupling constant' $G=g_{R}$ by the equations of motion (3.12), and thus the $\boldsymbol{V}_{S}$ may be called 'pre- $U(1)_{R}$ gauge multiplet'. In the present formulation, therefore, it is this pre$U(1)_{R}$ gauge multiplet $\boldsymbol{V}_{S}$ that we make couple to the hypermultiplet compensator at the beginning: namely, we have

$$
\mathcal{D}_{\mu} \mathcal{A}_{i}^{a}=\partial_{\mu} \mathcal{A}_{i}^{a}-V_{\mu i j} \mathcal{A}^{a j}-W_{S \mu}(i \vec{q} \cdot \vec{\sigma})^{a}{ }_{b} \mathcal{A}_{i}^{b}
$$

which reduces to the previous Eq. (3.14) after the equations of motion (3·12) are used. We assume that the pre- $U(1)_{R}$ gauge multiplet $\boldsymbol{V}_{S}$ does not have its own kinetic term; that is, $M_{S}$ is not contained in $\mathcal{N}(M)$. The kinetic term of the $U(1)_{R}$ gauge multiplet, of course, exists when $\operatorname{det} \mathcal{N}_{I J} \neq 0$ which we assume throughout this paper.

\section{$\S 4$. Compactifying on orbifold and the brane action}

We now compactify the fifth direction $y \equiv x^{4}$ on the orbifold $S^{1} / Z_{2}$, the two fixed planes of which are placed at $y=0$ and $y=\pi R \equiv \tilde{y}$. We must first know the properties of the fields under the $Z_{2}$ parity transformation, $y \rightarrow-y$. The parity quantum number $\Pi$ is defined by

$$
\Phi(-y)=\Pi(\Phi) \Phi(y)
$$

for boson fields $\Phi$, and, as discussed by BKVP, (1) by

$$
\psi^{i}(-y)=\Pi(\psi) \gamma_{5} M^{i}{ }_{j} \psi^{j}(y) \quad\left(\bar{\psi}^{i}(-y)=\Pi(\psi) M_{j}^{i} \bar{\psi}^{j}(y) \gamma_{5}\right)
$$

for $S U(2)$ spinor fermions $\psi^{i}(i=1,2)$. Consistency with the reality $\bar{\psi}^{i}=\left(\psi_{i}\right)^{\dagger} \gamma_{0}=\psi^{i \mathrm{~T}} C$ requires

$$
\left(M^{i}{ }_{j}\right)^{*}=\epsilon_{i k} M^{k}{ }_{l} \epsilon^{l j}, \quad \text { or } \quad M^{*}=-\sigma_{2} M \sigma_{2}
$$

and we can take

$$
M_{j}^{i}=\left(\sigma_{3}\right)_{j}^{i}=\delta_{j}^{i}(-1)^{j+1}
$$


without loss of generality. For fermion components $\zeta^{\alpha}(\alpha=1,2, \cdots, 2 r)$ of hypermultiplets, the parity is similarly defined by

$$
\zeta^{\alpha}(-y)=\Pi(\zeta) \gamma_{5} M_{\beta}^{\alpha} \zeta^{\beta}(y)
$$

and so the reality condition demands $\left(M^{\alpha}{ }_{\beta}\right)^{*}=\rho_{\alpha \gamma} M^{\gamma} \rho^{\delta \beta}$. Thus we can take $M^{\alpha}{ }_{\beta}=\sigma_{3} \otimes \mathbf{1}_{r}$ in the standard representation in which $\rho_{\alpha \beta}=\epsilon \otimes \mathbf{1}_{r}$.

The parity is determined by demanding the invariance of the action and the consistency of the both sides of the supersymmetry transformation rules. We find, for the Weyl multiplet fields and $Q$ - and $S$-transformation parameters $\varepsilon$ and $\eta$,

$$
\begin{aligned}
& \Pi\left(e_{\underline{\underline{a}}}^{\underline{a}}\right)=\Pi\left(e_{y}^{4}\right)=+1, \quad \Pi\left(e_{\underline{\mu}}^{4}\right)=\Pi\left(e \frac{a}{y}\right)=-1, \\
& \Pi\left(\psi_{\underline{\mu}}\right)=\Pi(\varepsilon)=+1, \quad \Pi\left(\psi_{y}\right)=\Pi(\eta)=-1, \\
& \Pi\left(b_{\underline{\mu}}\right)=\Pi\left(V_{\underline{\mu}}^{3}\right)=\Pi\left(V_{y}^{1,2}\right)=\Pi\left(v^{4 \underline{a}}\right)=\Pi(\chi)=\Pi(D)=+1, \\
& \Pi\left(b_{y}\right)=\Pi\left(V_{y}^{3}\right)=\Pi\left(V_{\underline{\mu}}^{1,2}\right)=\Pi\left(v^{\underline{a b}}\right)=-1,
\end{aligned}
$$

where the underlined indices $\underline{\mu}$ and $\underline{a}$ denote the four-dimensional parts of the five-dimensional curved index $\mu$ and flat index $a$. The fifth direction of $\mu$ and $a$ are denoted by $y$ and 4 , respectively. The (real) 'isovector' components $\vec{t}=\left(t^{1,2,3}\right)$ is generally defined for any symmetric $S U(2)$ tensor $t^{i j}$ (satisfying hermiticity $t^{i j}=\left(t_{i j}\right)^{*}$ ) as

$$
t_{j}^{i}\left(=t^{i k} \varepsilon_{k j}\right) \equiv i \vec{t} \cdot \vec{\sigma}_{j}^{i}
$$

For vector multiplet $\boldsymbol{V}=\left(M, W_{\mu}, \Omega^{i}, Y^{i j}\right)$, we have

$$
\begin{aligned}
& \Pi(M)=\Pi\left(W_{y}\right)=\Pi\left(Y^{1,2}\right)=\Pi_{\boldsymbol{V}}, \\
& \Pi(\Omega)=\Pi\left(W_{\underline{\mu}}\right)=\Pi\left(Y^{3}\right)=-\Pi_{\boldsymbol{V}} .
\end{aligned}
$$

We define the parity $\Pi_{\boldsymbol{V}}$ of vector multiplet $\boldsymbol{V}$ to be the parity $\Pi(M)$ of the first component scalar $M$. Normally the parity $\Pi_{\boldsymbol{V}}$ of vector multiplets must be +1 in five dimensions since they appear in the action via the homogeneous cubic function $\mathcal{N}(M)$ which should have even parity +1 . However, if a certain subset of the vector multiplets appear in $\mathcal{N}(M)$ only in the terms quadratic in them, then their parity assignment has a choice of \pm 1 .

For linear multiplet $\boldsymbol{L}=\left(L^{i j}, \varphi^{i}, E^{a}, N\right)$, we find

$$
\begin{aligned}
& \Pi\left(L^{1,2}\right)=\Pi(\varphi)=\Pi(N)=\Pi\left(E^{4}\right)=\Pi\left(E_{\underline{\mu} \underline{\underline{\rho}} \underline{\rho}}\right)=\Pi_{\boldsymbol{L}}, \\
& \Pi\left(L^{3}\right)=\Pi\left(E^{\underline{a}}\right)=\Pi\left(E_{y \underline{\mu} \underline{\underline{\nu}}}\right)=-\Pi_{\boldsymbol{L}} .
\end{aligned}
$$


The parity of the 4-form field $\left.H_{\mu \nu \rho \sigma}\right|_{V}$ also depends on the parity $\Pi_{\boldsymbol{V}}$ of the vector multiplet $\boldsymbol{V}$ used in the rewriting $N \rightarrow H_{\mu \nu \rho \sigma}$ :

$$
\Pi\left(H_{\underline{\mu} \underline{\underline{\nu} \underline{\rho}} \underline{\rho}}\right)=-\Pi_{\boldsymbol{V}} \Pi_{\boldsymbol{L}}, \quad \Pi\left(H_{\underline{y} \underline{\mu} \underline{\rho} \underline{\rho}}\right)=\Pi_{\boldsymbol{V}} \Pi_{\boldsymbol{L}}
$$

The hypermultiplet $\boldsymbol{H}^{\alpha}=\left(\mathcal{A}_{i}^{\alpha}, \zeta^{\alpha}, \mathcal{F}_{i}^{\alpha}\right)(\alpha=1,2, \cdots, 2 r)$ splits into $r$ pairs $\left(\boldsymbol{H}^{2 \hat{\alpha}-1}, \boldsymbol{H}^{2 \hat{\alpha}}\right)$ $(\hat{\alpha}=1,2, \cdots, r)$ in the standard representation in which $\rho_{\alpha \beta}=\epsilon \otimes \mathbf{1}_{r}$ and then the reality condition of the scalar components $\mathcal{A}^{\alpha}{ }_{i}, \epsilon^{i j} \mathcal{A}^{\beta}{ }_{j} \rho_{\beta \alpha}=\left(\mathcal{A}^{\alpha}{ }_{i}\right)^{*}$, implies that the $2 \times 2$ matrix $\left(\mathcal{A}^{2 \hat{\alpha}-1}{ }_{i}, \mathcal{A}^{2 \hat{\alpha}}{ }_{i}\right)$ with $i=1,2$ for each fixed $\hat{\alpha}$ (which can be identified with a quaterniont $)$ has the form

$$
\left(\begin{array}{cc}
\mathcal{A}^{2 \hat{\alpha}-1}{ }_{1} & \mathcal{A}^{2 \hat{\alpha}-1}{ }_{2} \\
\mathcal{A}^{2 \hat{\alpha}}{ }_{1} & \mathcal{A}^{2 \hat{\alpha}}{ }_{2}
\end{array}\right)=\mathcal{A}_{\hat{\alpha}}^{0} \mathbf{1}_{2}+\sum_{k=1}^{3} i \mathcal{A}_{\hat{\alpha}}^{k} \sigma_{k}=\left(\begin{array}{cc}
\mathcal{A}_{\hat{\alpha}}^{0}+i \mathcal{A}_{\hat{\alpha}}^{3} & i \mathcal{A}_{\hat{\alpha}}^{1}+\mathcal{A}_{\hat{\alpha}}^{2} \\
i \mathcal{A}_{\hat{\alpha}}^{1}-\mathcal{A}_{\hat{\alpha}}^{2} & \mathcal{A}_{\hat{\alpha}}^{0}-i \mathcal{A}_{\hat{\alpha}}^{3}
\end{array}\right) .
$$

with $\mathcal{A}_{\hat{\alpha}}^{0,1,2,3}$ being real. The components $\mathcal{F}_{\hat{\alpha}}^{0,1,2,3}$ are also defined similarly. Then, if the component $\mathcal{A}_{\hat{\alpha}}^{0}$ has a parity $\Pi_{\hat{\alpha}}$, then we have

$$
\Pi\left(\mathcal{A}_{\hat{\alpha}}^{0,3}\right)=\Pi\left(\mathcal{F}_{\hat{\alpha}}^{1,2}\right)=\Pi_{\hat{\alpha}}, \quad \Pi\left(\mathcal{A}_{\hat{\alpha}}^{1,2}\right)=\Pi\left(\zeta_{2 \hat{\alpha}-1,2 \hat{\alpha}}\right)=\Pi\left(\mathcal{F}_{\hat{\alpha}}^{0,3}\right)=-\Pi_{\hat{\alpha}}
$$

In the present gauged $U(1)_{R}$ supergravity, the vector multiplet $\boldsymbol{V}_{S}$ couples to the hypermultiplet compensator in the form (3·15). In order for the two terms $-V_{\mu i j} \mathcal{A}^{a j}$ and $-W_{S \mu}(i \vec{q} \cdot \vec{\sigma})^{a}{ }_{b} \mathcal{A}_{i}^{b}$ in the RHS of Eq. (3.15) to have the same $Z_{2}$ parity property, $\vec{q} \cdot \vec{\sigma}$ must commute or anti-commute with $\sigma_{3}$, and $\Pi\left(W_{S \underline{\mu}}\right)=+1$ when $\left[\vec{q} \cdot \vec{\sigma}, \sigma_{3}\right]=0$ and $\Pi\left(W_{S \underline{\mu}}\right)=-1$ when $\left\{\vec{q} \cdot \vec{\sigma}, \sigma_{3}\right\}=0$. This implies that the parity $\Pi_{S}$ of the vector multiplet $\boldsymbol{V}_{S}$ (i.e., $\Pi\left(M_{S}\right)$ ) should be

$$
\Pi_{S}= \begin{cases}-1 & \text { when } \vec{q} \cdot \vec{\sigma}=\sigma_{3} \\ +1 & \text { when } \vec{q} \cdot \vec{\sigma}=\sigma_{1} \cos \theta+\sigma_{2} \sin \theta\end{cases}
$$

In order for the 4-form field action $\mathcal{L}_{\mathrm{VL}}\left(\boldsymbol{V}_{S},\left.\boldsymbol{L}\right|_{\boldsymbol{V}_{R}}\right)$ in Eq. (3.9) to be invariant under $Z_{2}$-parity, we must have $\Pi_{S} \Pi_{L}=+1$.

As discussed by BKVP, we want to have the pullback of the component $H_{\underline{\mu} \underline{\nu} \underline{\rho} \underline{\sigma}}$ on the brane nonvanishing so that $\Pi\left(H_{\underline{\mu} \underline{\nu} \underline{\rho} \underline{\sigma}}\right)=+1$. This implies $\Pi_{R} \Pi_{\boldsymbol{L}}=-1$ and hence $\Pi_{R}=-\Pi_{S}$ and $\Pi(G)=-1$.

We allow the cubic term in $M_{R}$ to exist in $\mathcal{N}(M)$, thus allowing the Chern-Simons term of the gauge field $W_{R \mu}$ in the five dimensional bulk, we assign $\Pi_{R}=+1$, then we have $\Pi_{S}=-1$ and $\Pi_{\boldsymbol{L}}=-1$. Note that, since $\Pi_{S}=-1$, the vector multiplet $\boldsymbol{V}_{S}$ or its first component $M_{S}$ can appear at most only in quadratic form in $\mathcal{N}(M)$. For simplicity, we assume that $\boldsymbol{V}_{S}$ does not appear in $\mathcal{N}(M)$ at all, as announced before.

\footnotetext{
* Quaternion $\boldsymbol{q}=\mathcal{A}_{\hat{\alpha}}^{0}-\boldsymbol{i} \mathcal{A}_{\hat{\alpha}}^{1}-j \mathcal{A}_{\hat{\alpha}}^{2}-\boldsymbol{k} \mathcal{A}_{\hat{\alpha}}^{3}$
} 
With these parity quantum numbers kept in mind, we consider the transformation rules $(3 \cdot 7)$ of $H_{\mu \nu \rho \sigma}$ and

$$
\delta M=2 i \bar{\varepsilon} \Omega, \quad \delta L^{i j}=2 i \bar{\varepsilon}^{(i} \varphi^{j)},
$$

for the first scalar components $M$ and $L^{i j}$ of vector and linear multiplets. Keeping all the even parity fields nonvanishing on the brane in the RHSs of these equations, we find the following transformation rules on the brane:

$$
\begin{aligned}
& \delta H_{\underline{\mu} \underline{\rho} \underline{\rho} \underline{\sigma}}=4 \partial_{[\underline{\mu}} \Lambda_{\underline{\nu} \underline{\rho} \underline{\sigma}]}+2 i \overline{\bar{\varepsilon}} \gamma_{\underline{\mu} \underline{\underline{\nu}} \underline{\rho} \underline{\sigma}} \varphi M_{R} \\
& -4 \bar{\varepsilon} \gamma_{\underline{\mu} \underline{\underline{\rho} \underline{\rho} \underline{\sigma}}} \gamma_{5} \Omega_{R} L^{3}+16 \bar{\varepsilon} \gamma_{5} \gamma_{[\underline{\mu} \underline{\mu} \underline{\rho}} \psi_{\underline{\sigma}]} M_{R} L^{3} \text {, } \\
& \delta M_{\mathrm{R}}=2 i \bar{\varepsilon} \Omega_{R}, \quad \delta L^{3}=\bar{\varepsilon} \gamma_{5} \varphi .
\end{aligned}
$$

Here we have used an identity $\bar{\varepsilon} \gamma_{5} \varphi=-2 \bar{\varepsilon}^{(1} \varphi^{2)}$ which holds on the brane for $S U(2)$ Majorana spinors $\varepsilon^{i}$ and $\varphi^{i}$ with $\Pi(\varepsilon)=+1$ and $\Pi(\varphi)=-1$. Now it is clear that the following brane action is superconformal invariant:

$$
S_{\text {brane }}=\int d^{5} x\left(\Lambda_{1} \delta(y)+\Lambda_{2} \delta(y-\tilde{y})\right)\left[\frac{1}{4 !} \epsilon^{\mu \nu \rho \sigma y} H_{\mu \nu \rho \sigma}+2 e_{(4)} M_{\mathrm{R}} L^{3}\right]
$$

where $e_{(4)} \equiv e / e_{y}^{4}$ is the determinant of the four dimensional vierbein on the brane.

\section{$\S 5$. Relation between bulk cosmological constant and brane tensions}

Now the action of our total system is given by the bulk action $\mathcal{L}_{0}$ [Eq. (2.4)] plus the 4-form field action $\mathcal{L}_{4 \text {-form }}\left[\right.$ Eq. (3.9)] plus the brane action $\mathcal{L}_{\text {brane }}$ [Eq. (4·16)]. The latter two parts $\mathcal{L}_{4 \text {-form }}+\mathcal{L}_{\text {brane }}$ give an off-shell generalization of the BKVP action $\mathbb{\text { U }}$ ) for realizing the odd parity coupling 'constant' (field) $G(x)$.

Indeed, variation of the components $\varphi^{i}$ and $E_{\mu \nu \rho}$ of the linear multiplet still gives the same equations as in Eq. (3.12): $\Omega_{S j}=G \Omega_{R j}$ and $F_{\lambda \mu}\left(W_{S}\right)=G F_{\lambda \mu}\left(W_{R}\right)$. The latter implies that $W_{S}^{\mu}=G W_{R}^{\mu}$ up to a gauge transformation if $G$ is a constant. This equation clearly shows that the field $G(x)$ plays the role of $U(1)_{R}$ gauge coupling constant. On the other hand, the equation obtained by variation of the 4-form field $H_{\mu \nu \rho \sigma}$ is now changed in the presence of branes into

$$
\partial_{\mu} G=2 \delta_{\mu}^{y}\left(\Lambda_{1} \delta(y)+\Lambda_{2} \delta(y-\tilde{y})\right)
$$

The integrability condition of this equation on the orbifold $S^{1} / Z_{2}$ demands the condition

$$
\Lambda_{1}=-\Lambda_{2} \equiv g_{R}
$$


and then the solution of $G(y)$ is given by

$$
G=g_{R} \epsilon(y)
$$

with a periodic sign function $\epsilon(y)$ (with period $2 \tilde{y}$ ) which is defined as

$$
\epsilon(y) \equiv\left\{\begin{array}{ll}
+1 & \text { for } 0<y<\tilde{y} \\
-1 & \text { for }-\tilde{y}<y<0 \\
0 & \text { for } y=0, \tilde{y}
\end{array} .\right.
$$

That is, the coupling 'constant' $G$ changes its sign across the branes and is really constant away from the branes.

Let us now discuss the relation between the cosmological constant in 5D bulk and the brane tensions of the boundary planes. First note that the auxiliary fields $Y^{i j}$ are contained in the action in the form [See $(2 \cdot 7)$ and $(3 \cdot 9)]$

$$
-\frac{1}{2} \mathcal{N}_{I J} Y_{i j}^{I} Y^{J i j}+Y_{i j}^{I} \mathcal{Y}_{I}^{i j}+Y_{i j}^{S} \mathcal{A}_{a}^{i}\left(2 g t_{S}\right)^{a b} \mathcal{A}_{b}^{j}+\left(Y^{S}-G Y^{R}\right)_{i j} L^{i j}
$$

where $a, b=1,2$ are the indices of the first compensator $\mathcal{A}_{i}^{a}$ only to which the vector multiplet $\boldsymbol{V}_{S}$ couples, $\left(g t_{S}\right)^{a}{ }_{b}=\left(i \sigma_{3}\right)^{a}{ }_{b}$ and the last term came from $\mathcal{L}_{4 \text {-form. }}$. The vector multiplet $\boldsymbol{V}_{S}$ is special since it is not contained in $\mathcal{N}(M)$ and so has no kinetic term. Then the auxiliary field component $Y_{S}$ appears in the action only linearly and plays a role of Lagrange multiplier yielding a constraint equation:

$$
\begin{aligned}
& L^{i j}=-2 \mathcal{A}_{a}^{i}\left(i \sigma_{3}\right)^{a b} \mathcal{A}_{b}^{j} \equiv L_{\text {sol }}^{i j}, \quad \text { or equivalently } \\
& L^{1}=L^{2}=0 \quad \text { and } \quad L^{3}=-2 i \mathcal{A}_{a}^{1}\left(i \sigma_{3}\right)^{a b} \mathcal{A}_{b}^{2} \equiv L_{\mathrm{sol}}^{3}
\end{aligned}
$$

Then the Eq. (5.5) is rewritten into

$$
\begin{gathered}
-\frac{1}{2} \mathcal{N}_{I J}\left(Y-Y_{\mathrm{sol}}^{\prime}\right)_{i j}^{I}\left(Y-Y_{\mathrm{sol}}^{\prime}\right)^{J i j}+\frac{1}{2} \mathcal{N}_{I J} Y_{\mathrm{sol} i j}^{\prime I} Y_{\mathrm{sol}}^{\prime J j} \\
Y_{\mathrm{sol}}^{\prime I i j} \equiv\left(\mathcal{N}^{-1}\right)^{I J}\left(\mathcal{Y}_{J}^{i j}-G V_{J} L_{\mathrm{sol}}^{i j}\right)
\end{gathered}
$$

After the auxiliary fields $Y_{S}, \varphi^{i}, E_{\mu \nu \rho}, H_{\mu \nu \rho \sigma}$ and $Y^{I} \mathrm{~S}$ are eliminated, the brane action now takes the form

$$
S_{\text {brane }}=-g_{R} \int d^{5} x(\delta(y)-\delta(y-\tilde{y}))\left[3 e_{(4)} W L_{\text {sol }}^{3}\right],
$$

where $W \equiv-(3 / 2) M_{R}=-(3 / 2) V_{I} M^{I}$ is the 'superpotential' introduced later in Eq. (5·15) below. This shows that brane tensions of the planes at $y=0$ and $y=\tilde{y}$ are given by $\mp 3 g_{R} W L_{\text {sol }}^{3}$, respectively.

* We adopt the convention that the usual expression for the Pauli matrix $\vec{\sigma}$ applies to the matrix with index position $\vec{\sigma}^{i}{ }_{j}$. The indices $i$ and $j$ are raised or lowered by using $\epsilon$ tensor, so that, for instance, $\vec{\sigma}^{i j}=\epsilon^{j k} \vec{\sigma}^{i}{ }_{k}=-\vec{\sigma}^{i}{ }_{k} \epsilon^{k j}$ denotes $-\vec{\sigma} \epsilon$ as a matrix. 
Next we turn to compute the scalar potential in 5D bulk. For that purpose, it is better to discuss separately two cases of one and two compensators. As noted before, the hypermultiplet scalars $\mathcal{A}_{i}^{\alpha}(\alpha=1,2, \cdots, 2 r)$ (or fermions $\left.\zeta^{\alpha}\right)$ have the metric matrix $d_{\alpha}{ }^{\beta}$ given in $2 \cdot 6$ ) in their kinetic term, and so the first $2 p$ components $\mathcal{A}_{i}^{\alpha}$ with $\alpha=1,2, \cdots, 2 p$, corresponding to $p$ quaternions, have negative metric and are called compensators, while the rest components $\mathcal{A}_{i}^{\underline{\alpha}}$ which we denote with underlined index $\underline{\alpha}$ have positive metric and represent usual matter fields. We consider the simplest two cases of one $(p=1)$ and two $(p=2)$ compensators, separately.

\section{1. $p=1$ case}

First is the most common $p=1$ case, containing a single compensating hypermultiplet $\boldsymbol{H}^{a}$ with $a=1,2$. Independently of $p$, we always fix the $S U(2)$ gauge by imposing the condition

$$
\mathcal{A}_{i}^{a}(x)=a(x) \delta_{i}^{a}, \quad a(x): \text { real positive }
$$

on the first compensator scalars $\mathcal{A}_{i}^{a=1,2}$, and so the $L_{\text {sol }}^{3}$ in Eq. (5.6) takes the form,

$$
L_{\mathrm{sol}}^{3}=-2 i \mathcal{A}_{a}^{1}\left(i \sigma_{3}\right)^{a b} \mathcal{A}_{b}^{2}=-2\left(\sigma_{3}\right)^{1}{ }_{1} a^{2}(x)=-2 a^{2}(x) .
$$

In the present case of $p=1$, the equation of motion $\mathcal{A}^{2}+2=0$ for $\mathcal{A}^{2} \equiv \mathcal{A}_{i}^{\alpha} d_{\alpha}{ }^{\beta} \mathcal{A}_{\beta}^{i}=$ $-\left|\mathcal{A}_{i}^{a}\right|^{2}+\left|\mathcal{A}_{i}^{\underline{\alpha}}\right|^{2}$ determines the magnitude $a(x)$ to be

$$
a(x)=\sqrt{1+\frac{1}{2}\left|\mathcal{A}_{i}^{\underline{\alpha}}\right|^{2}} .
$$

The scalar potential in the bulk is given by

$$
\begin{aligned}
V & =-\left.\frac{1}{2} \mathcal{N}_{I J} Y_{\text {solij }}^{\prime I} Y_{\text {sol }}^{\prime J i j}\right|_{\text {bosonic }}-\mathcal{A}_{i}^{\bar{\alpha}}(g M)^{2}{ }_{\alpha}{ }^{\beta} \mathcal{A}_{\beta}^{i} \\
& =\left(a^{I J}-M^{I} M^{J}\right) P_{I}^{i j}\left(P_{J}^{i j}\right)^{*}-\left|Q_{i}^{a}\right|^{2}+\left|Q_{i}^{\underline{\alpha}}\right|^{2}
\end{aligned}
$$

where use has been made of $\left(\mathcal{N}^{-1}\right)^{I J}=-(1 / 2)\left(a^{I J}-M^{I} M^{J}\right)$ with $a^{I J}$ denoting the inverse of $a_{I J}$ in Eq. (2.5), and taking Eq. (5·7) into account,

$$
\begin{aligned}
P_{I}^{i j} & \equiv-\frac{1}{2} G V_{I} L_{\text {sol }}^{i j}+\left.\frac{1}{2} \mathcal{Y}_{I}^{i j}\right|_{\text {bosonic }}=G V_{I} a^{2}\left(i \sigma_{3}\right)^{i j}+\mathcal{A}_{\underline{\alpha}}^{(i}\left(g t_{I}\right)^{\underline{\alpha} \underline{\beta}} \mathcal{A}_{\underline{\beta}}^{j)}, \\
Q_{i}^{a} & \equiv \delta_{G}(M) \mathcal{A}_{i}^{a}=M_{S}\left(g t_{S}\right)^{a}{ }_{b} \mathcal{A}_{i}^{b}=G M_{R}\left(i \sigma_{3}\right)^{a}{ }_{b} \mathcal{A}_{i}^{b}=G V_{I} M^{I} a\left(i \sigma_{3}\right)^{a}{ }_{i}, \\
Q_{i}^{\underline{\alpha}} & \equiv \delta_{G}(M) \mathcal{A}_{i}^{\underline{\alpha}}=M^{I}\left(g t_{I}\right)^{\underline{\alpha}}{ }_{\underline{\beta}} \mathcal{A}_{i}^{\underline{\beta}} .
\end{aligned}
$$

* Note that the four compensating bosons $\mathcal{A}_{i}^{a}(a=1,2, i=1,2)$ carrying negative metric in this $p=1$ case are eliminated by the three gauge-fixing conditions of $S U(2),(5 \cdot 9)$, and this equation of motion $\mathcal{A}^{2}=-2 \mathcal{N}$ with dilatation gauge condition $\mathcal{N}=1$. The target manifold spanned by the hypermultiplet scalars constrained by these conditions becomes $U S p(2,2 q) / U S p(2) \times U S p(2 q) .14)$ 
If the hypermultiplet fields $\mathcal{A}_{i}^{\underline{\alpha}}$ other than the compensator $\mathcal{A}_{i}^{a}$ are assumed not to be charged, i.e., $g t_{I}=0$, then the potential reduces to a simple form

$$
V=\frac{3}{2} g_{R}^{2}\left\{3|a|^{4} g^{x y} \frac{\partial W}{\partial \varphi^{x}} \frac{\partial W}{\partial \varphi^{y}}-\left(|a|^{4}+3|a|^{2}\right) W^{2}\right\} .
$$

Here $\varphi^{x}(x=1, \cdots, n)$ are independent $n$ scalar fields with which $n+1$ vector multiplet scalars $M^{I}$, constrained by $\boldsymbol{D}$ gauge condition $\mathcal{N}(M)=1$, are parametrized. 8 ) We have used the relations $a^{I J}=g^{x y} h_{x}^{I} h_{y}^{J}+h^{I} h^{J}$ and $h^{I}=-\sqrt{2 / 3} M^{I}$ given in I, Eqs. (I $7 \cdot 3$ ) and (I $7 \cdot 1$ ), and the definitions

$$
W \equiv \sqrt{\frac{2}{3}} V_{I} h^{I}=-\frac{2}{3} V_{I} M^{I}, \quad \frac{\partial W}{\partial \varphi^{x}}=-\frac{2}{3} V_{I} h_{x}^{I}=-\frac{2}{3} V_{I} M_{, x}^{I} .
$$

If the system contains no physical vector multiplets (i.e., $n=0$ ) so that there appear no scalars $\varphi^{x}$ and only the graviphoton with $I=0$ exists as a vector field, then, the "superpotential' $W$ reduces to a constant and the bulk scalar potential $V$ to $V=-(3 / 2) g_{R}^{2}\left(|a|^{4}+\right.$ $\left.3|a|^{2}\right) W^{2}$. This should be compared with the brane tensions $\pm 6 g_{R} a W$ of the planes at $y=0$ and $\tilde{y}$. If the system has no matter-hypermultiplets either, i.e., $q=0$, then, $a$ becomes 1 and the bulk scalar potential further reduces to $V=-6 g_{R}^{2} W^{2}$ and brane tensions to $\pm 6 g_{R} W$, yielding exactly the same relations required by the Randal and Sundrum.1)

\section{2. $p=2$ case}

Next we turn to $p=2$ case, for which the manifold spanned by the hypermultiplet scalar fields becomes $S U(2, q) / S U(2) \times S U(q) \times U(1)$ and, when $q=1$, this just corresponds to the manifold of the universal hypermultiplet 15 appearing in the reduction of the heterotic M-theory on $S^{1} / Z_{2}$ to five dimensions. (6) In this $p=2$ case, as explained in I in detail, we need to introduce another special vector multiplet $\boldsymbol{V}_{T}$ which possesses no kinetic term either and couples to the hypermultiplets via charge $T=\sigma_{3} \otimes \mathbf{1}_{2+q}$, that is, the hypermultiplet $\boldsymbol{H}^{\alpha}$ with odd (even) $\alpha$ carries $+1(-1)$ charge. In this case the auxiliary field component $Y_{T}^{i j}$ again appears in the action as a multiplier field and gives three constraints

$$
\mathcal{A}_{a i}\left(\sigma_{3}\right)^{a}{ }_{b} \mathcal{A}_{j}^{b}+\mathcal{A}_{a^{\prime} i}\left(\sigma_{3}\right)^{a^{\prime}}{ }_{b^{\prime}} \mathcal{A}_{j}^{b^{\prime}}=\mathcal{A}_{\underline{\alpha} i}\left(\sigma_{3}\right)^{\underline{\alpha}}{ }_{\underline{\beta}} \mathcal{A}_{j}^{\underline{\beta}}
$$

where the primed indices $a^{\prime}, b^{\prime}=3,4$ are used to denote those of the second compensator $\mathcal{A}_{i}^{a^{\prime}}$ and the indices $\alpha, \beta$ of $\left(\sigma_{3}\right)^{\alpha}{ }_{\beta}$ should generally be understood to be 1 and 2 when they are odd and even, respectively. The equation of motion $\mathcal{A}^{2} \equiv \mathcal{A}_{i}^{\alpha} d_{\alpha}{ }^{\beta} \mathcal{A}_{\beta}^{i}=-2$ gives

$$
-\left|\mathcal{A}_{i}^{a}\right|^{2}-\left|\mathcal{A}_{i}^{a^{\prime}}\right|^{2}+\left|\mathcal{A}_{i}^{\underline{\alpha}}\right|^{2}=-2
$$


If we impose the $S U(2)$ gauge-fixing condition Eq. (5.9) and $\mathcal{A}^{a^{\prime}=3}{ }_{i=2}=$ real as the $U(1)_{T}$ gauge-fixing, the solution of these constraints (5.16) and (5.17) is shown in the Appendix to be given in terms of two $q$-component complex vectors $\phi_{1}$ and $\phi_{2}$ as

$$
\begin{aligned}
& \mathcal{A}^{a=1}{ }_{i=1}=\sqrt{\frac{1-\left|\phi_{2}\right|^{2}}{2\left(1-\left|\phi_{1}\right|^{2}-\left|\phi_{2}\right|^{2}+\left|\phi_{1}\right|^{2}\left|\phi_{2}\right|^{2}-\left|\phi_{1}^{\dagger} \cdot \phi_{2}\right|^{2}\right)}} \equiv a, \\
& \mathcal{A}^{a^{\prime}=3}{ }_{i=1}=a \frac{\phi_{2}^{\dagger} \cdot \phi_{1}}{1-\left|\phi_{2}\right|^{2}}, \quad \mathcal{A}^{a^{\prime}=3}{ }_{i=2}=\sqrt{\frac{1}{2\left(1-\left|\phi_{2}\right|^{2}\right)}} \equiv b, \\
& \mathcal{A}^{\mathcal{A}^{\underline{\alpha}=\text { odd }_{i=1}}=a\left(\phi_{1}+\frac{\phi_{2}^{\dagger} \cdot \phi_{1}}{1-\left|\phi_{2}\right|^{2}} \phi_{2}\right), \quad \mathcal{A}^{\underline{\alpha}^{\alpha}={ }_{i=2}}{ }_{i=2}=b \phi_{2},}
\end{aligned}
$$

where $|\phi|^{2} \equiv \phi^{\dagger} \cdot \phi$ and we have shown only the components $\mathcal{A}_{i}^{\alpha}$ with odd $\alpha=2 \hat{\alpha}-1$, whose complex conjugates essentially give the even $\alpha=2 \hat{\alpha}$ components, $\mathcal{A}^{2 \hat{\alpha}_{i}}=\left(\mathcal{A}^{2 \hat{\alpha}-1}{ }_{j}\right)^{*} \epsilon_{j i}$, because of the quaternionic nature (1.11) of the hypermultiplet scalars $\mathcal{A}_{i}^{\alpha}$.

The scalar potential in the bulk is now given by

$$
V=\left(a^{I J}-M^{I} M^{J}\right) P_{I}^{i j}\left(P_{J}^{i j}\right)^{*}-\left|Q_{i}^{a}\right|^{2}-\left|Q_{i}^{a^{\prime}}\right|^{2}+\left|Q_{i}^{\underline{\alpha}}\right|^{2},
$$

where $P_{I}^{i j}$ is the same as before while $Q_{i}^{\alpha}$ are now

$$
\begin{aligned}
Q_{i}^{a} & \equiv \delta_{G}(M) \mathcal{A}_{i}^{a}=\left(M_{S}+M_{T}\right)\left(i \sigma_{3}\right)^{a}{ }_{b} \mathcal{A}_{i}^{b}=\left(G V_{I} M^{I}+M_{T}\right) a\left(i \sigma_{3}\right)^{a}{ }_{i}, \\
Q_{i}^{a^{\prime}} & \equiv \delta_{G}(M) \mathcal{A}_{i}^{a^{\prime}}=M_{T}\left(i \sigma_{3}\right)^{a^{\prime}}{ }_{{ }^{\prime}} \mathcal{A}_{i}^{b^{\prime}}, \\
Q_{i}^{\underline{\alpha}} & \equiv \delta_{G}(M) \mathcal{A}_{i}^{\underline{\alpha}}=\left(M_{T}\left(i \sigma_{3}\right)^{\underline{\underline{\alpha}}} \underline{\underline{\beta}}+M^{I}\left(g t_{I}\right)^{\underline{\alpha}} \underline{\underline{\beta}}\right) \mathcal{A}_{i}^{\underline{\beta}} .
\end{aligned}
$$

If we assume again that the hypermultiplet matter fields $\mathcal{A}_{i}^{\underline{\alpha}}$ other than the compensators $\mathcal{A}_{i}^{a}$ and $\mathcal{A}_{i}^{a^{\prime}}$ are not charged, i.e., $g t_{I}=0$, then the potential takes a simpler form. The contribution of the three $\left|Q_{i}^{\alpha}\right|^{2}$ terms is evaluated as

$$
\begin{aligned}
& -\left|Q_{i}^{a}\right|^{2}-\left|Q_{i}^{a^{\prime}}\right|^{2}+\left|Q_{i}^{\underline{\alpha}}\right|^{2} \\
& \quad=-2\left(M_{S}+M_{T}\right)^{2}|a|^{2}-M_{T}^{2}\left|\mathcal{A}_{i}^{a^{\prime}}\right|^{2}+M_{T}^{2}\left|\mathcal{A}_{i}^{\underline{\alpha}}\right|^{2} \\
& \quad=-2 M_{S}^{2}|a|^{2}-4 M_{S} M_{T}|a|^{2}+M_{T}^{2}\left(-\left|\mathcal{A}_{i}^{a}\right|^{2}-\left|\mathcal{A}_{i}^{a^{\prime}}\right|^{2}+\left|\mathcal{A}_{i}^{\underline{\alpha}}\right|^{2}\right) \\
& \quad=-2 M_{S}^{2}|a|^{2}-4 M_{S} M_{T}|a|^{2}-2 M_{T}^{2}
\end{aligned}
$$

where we have inserted the Eq. (5.17) in the last step. Since $\boldsymbol{V}_{T}$ has no its own kinetic term and the scalar component $M_{T}$ appears only here in the action, so that $M_{T}$ can be eliminated by using its equation of motion $M_{T}=-M_{S}|a|^{2}$. Then this contribution (5.22) reduces to

$$
-2 M_{S}^{2}|a|^{2}+2 M_{S}^{2}|a|^{4}
$$


The first term of this is the same contribution as the previous $p=1$ case, which together with the first $\left|P_{I}^{i j}\right|^{2}$ term gives the same expression as the previous potential (5·14) (although $a$ here is given by Eq. (5.18) and different from the previous one (5.11)). Thus the second term is the additional new contribution in this $p=2$ case,

$$
2 M_{S}^{2}|a|^{4}=2 g_{R}^{2}\left(V_{I} M^{I}\right)^{2}|a|^{4}=\frac{9}{2} g_{R}^{2} W^{2}|a|^{4} .
$$

Adding this to the previous potential (5·14), we find the scalar potential in $p=2$ case as

$$
V=\frac{3}{2} g_{R}^{2}\left\{3|a|^{4} g^{x y} \frac{\partial W}{\partial \varphi^{x}} \frac{\partial W}{\partial \varphi^{y}}-\left(3|a|^{2}-2|a|^{4}\right) W^{2}\right\} .
$$

Again consider the special case that the system contains no physical vector multiplets and so no scalars $\varphi^{x}$, then, $W$ is constant. Moreover, consider the case $q=1$; that is, the system contains only a single physical hypermultiplet and corresponds to the universal hypermultiplet. Then, $\phi_{1}$ and $\phi_{2}$ are single component complex fields and can be rewritten in terms of more popular variables, a complex $\xi$ and real $V$ and $\sigma:$ (i6)

$$
\phi_{1}=\frac{2 \xi}{1+S}, \quad \phi_{2}=\frac{1-S}{1+S}, \quad S \equiv V+\bar{\xi} \xi+i \sigma .
$$

Then, $a$ in Eq. $(5 \cdot 18)$ reduces to

$$
a=\sqrt{\frac{1}{2}+\frac{|\xi|^{2}}{2 V}} .
$$

We can easily see that the result (5.25) with this $a$ in (5.27) is easily seen to agree with the result by Falkowski, Lalak and Pokorski. 4 - Actually the present result (5.25) reproduces only a part of their result, the part proportional to their $\beta$ parameter, but the part proportional to their $\alpha$ parameter is missing. The reason is clear. Up to here we have tacitly assumed that our pre- $U(1)_{R}$ gauge multiplet $\boldsymbol{V}_{S}$ couples only to the first compensator. Namely the $U(1)_{S}$ charge $g t_{S}$ to which $\boldsymbol{V}_{S}$ couples has been chosen to be

$$
g t_{S}=i\left(\begin{array}{ccc}
1 & 0 & 0 \\
0 & 0 & 0 \\
0 & 0 & 0
\end{array}\right) \quad \text { on } \quad \mathcal{A}_{\text {odd }} \equiv\left(\mathcal{A}^{2 \hat{\alpha}-1_{i}}\right) \equiv\left(\begin{array}{c}
\mathcal{A}^{a=1}{ }_{i} \\
\mathcal{A}^{a^{\prime}={ }_{i}} \\
\mathcal{A}^{\underline{\alpha}={ }_{i}}{ }_{i}
\end{array}\right) \text {. }
$$

However, the isometry group of the hypermultiplet manifold is $U(2, q=1)$, which is given by the $3 \times 3$ matrices $U_{2,1}$ acting on this $3 \times 2$ matrix $\mathcal{A}_{\text {odd }}$ from left (and its complex conjugate $\left(U_{2,1}\right)^{*}$ on the even row elements $\left.\mathcal{A}_{\text {even }} \equiv\left(\mathcal{A}^{2 \hat{\alpha}_{i}}\right)\right)$. When the system is compactified on $S^{1} / Z_{2}$, the isometry group is reduced to the subgroup $U(1) \times U(1, q=1)$ since it should commute with the $Z_{2}$ parity transformation under which $\left(\mathcal{A}^{a=1}{ }_{1}, \mathcal{A}^{a^{\prime}=3}{ }_{1}, \mathcal{A}^{\underline{\alpha}=5}{ }_{1}\right)$ get signs $(+1,-1,-1)$. (This parity assignment corresponds to $\Pi\left(\phi_{1}\right)=-1$ and $\Pi\left(\phi_{2}\right)=+1$ (or, 
$\Pi(\xi)=-1$ and $\Pi(S)=+1$ ) and is consistent with Eq. (4.12) and expressions of $\mathcal{A}^{2 \hat{\alpha}-i}{ }_{i}$ in Eqs. (5.18) and (5.19).) The $U(1)_{S}$ charge $g t_{S}$ to which $\boldsymbol{V}_{S}$ couples can actually be any $U(1)$ generator of this isometry group $U(1) \times U(1, q=1)$. The $U(1)_{S}$ generator which was chosen by Falkowski et al is given, in our terminology, by

$$
g t_{S}=i \alpha\left(\begin{array}{ccc}
0 & 0 & 0 \\
0 & -1 & -1 \\
0 & 1 & 1
\end{array}\right)+i \beta\left(\begin{array}{ccc}
1 & 0 & 0 \\
0 & 0 & 0 \\
0 & 0 & 0
\end{array}\right)
$$

The first generator with coefficient $\alpha$ corresponds to the isometry in $U(1, q=1)$ shifting the field $\sigma=\operatorname{Im} S$ in Eq. (5.26) and the second generator with coefficient $\beta$ is the original $U(1)$ charge in Eq. (5.28). Also in this case, one can compute the bulk cosmological constant and brane tensions in the same way as above. It is convenient to use the $3 \times 2$ matrix $\Phi \equiv \mathcal{A}_{\text {odd }}$ consisting of the odd row elements (5.28) and notation defined in Appendix, with which we find formulas $\left(W_{, x} \equiv \partial W / \partial \varphi^{x}\right)$

$$
\begin{aligned}
& L_{\mathrm{sol}}^{i}{ }_{j}=4\left(\Phi^{\dagger} \eta\left(g t_{S}\right) \Phi-\frac{1}{2} \mathbf{1}_{2} \operatorname{tr}\left(\Phi^{\dagger} \eta g t_{S} \Phi\right)\right)^{i}{ }_{j} \\
& \left(a^{I J}-M^{I} M^{J}\right) P_{I}^{i j}\left(P_{J}^{i j}\right)^{*}=\frac{3}{16} g_{R}^{2}\left(3 g^{x y} W_{, x} W_{, y}-W^{2}\right) \operatorname{tr}\left(L_{\mathrm{sol}}^{\dagger} L_{\mathrm{sol}}\right) \\
& -\left|Q_{i}^{a}\right|^{2}-\left|Q_{i}^{a^{\prime}}\right|^{2}+\left|Q_{i}^{\underline{\alpha}}\right|^{2}=-2 M_{S}^{2}\left\{\operatorname{tr}\left(\Phi^{\dagger} \eta\left(g t_{S}\right)^{2} \Phi\right)+\left[\operatorname{tr}\left(\Phi^{\dagger} \eta g t_{S} \Phi\right)\right]^{2}\right\} \\
& +2\left(M_{T}+i M_{S} \operatorname{tr}\left(\Phi^{\dagger} \eta g t_{S} \Phi\right)\right)^{2}
\end{aligned}
$$

The last perfect square term vanishes if we use $M_{T}$ equation of motion. Inserting the expression (5.29) for the $U(1)_{S}$ generator $g t_{S}$ in this case and expressions $(5 \cdot 18)$ and $(5 \cdot 19)$ for $\Phi=\mathcal{A}_{\text {odd }}$, and using $M_{S}^{2}=(9 / 4) g_{R}^{2} W^{2}$ and parametrization (5.26), it is straightforward to find

$$
\begin{aligned}
L_{\mathrm{sol}}^{3}= & -\beta\left(1+\frac{|\xi|^{2}}{V}\right)-\frac{\alpha}{V}\left(\frac{V-|\xi|^{2}}{V+|\xi|^{2}}\right) \\
V= & \frac{9}{2} g_{R}^{2} g^{x y} \frac{\partial W}{\partial \varphi^{x}} \frac{\partial W}{\partial \varphi^{y}}\left\{\frac{1}{4}\left[\beta\left(1+\frac{|\xi|^{2}}{V}\right)-\frac{\alpha}{V}\right]^{2}+\frac{\alpha \beta}{V}\right\} \\
& +\frac{3}{2} g_{R}^{2} W^{2}\left\{\frac{1}{2}\left[\beta\left(1+\frac{|\xi|^{2}}{V}\right)-\frac{\alpha}{V}\right]^{2}-\frac{3}{2} \beta^{2}\left(1+\frac{|\xi|^{2}}{V}\right)-\frac{\alpha \beta}{V}\right\} .
\end{aligned}
$$

We see that the brane tension $\mp 3 g_{R} W L_{\text {sol }}^{3}$ with this $L_{\text {sol }}^{3}$ coincides with Falkowski et al's 4 provided that $\xi$ terms are eliminated by assigning odd parity to $\xi$. Their tension parameter $\Lambda$ is identified with our $3 g_{R} W$. If the system is reduced to the $n=0$ case where $W$ is constant, then this scalar potential $V$ also agrees with theirs aside from the term proportional to $\alpha \beta$, which we believe is their error or typo. 


\section{$\S 6 . \quad$ Discussions}

We have given an off-shell formulation of the odd-parity 'coupling constant' field $G(x)$ and 4-form multiplier field $H_{\mu \nu \rho \sigma}$. This was achieved by rewriting a neutral linear multiplet $\boldsymbol{L}$ in terms of 3-form and 4-form gauge fields. In particular, we need a vector multiplet background in rewriting the auxiliary scalar component $N$ of $\boldsymbol{L}$ into the 4 -form gauge field $H_{\mu \nu \rho \sigma}$, and we obtain the 4-form field action in the five-dimensional bulk by applying the invariant action formula for the product of an Abelian vector multiplet $\boldsymbol{V}$ and the linear multiplet $\boldsymbol{L}$. Using here a vector multiplet different from the above background vector multiplet, we can obtain the 'coupling constant' field $G(x)$ as the ratio of the two scalar components $M$ of the these two vector multiplets. All the components of this linear multiplet $\boldsymbol{L}$ now become Lagrange multiplier fields and, in particular, the 4-form gauge field component becomes the multiplier demanding that $G$ be a constant and change sign across the branes.

We have presented this formulation in a rather general system of Yang-Mills and hypermultiplet matters and discussed the relation between the bulk cosmological constant and the brane tensions of two boundary planes. The result agreed with the other authors' ones when the system is reduced to some special cases.

It is interesting that our approach suggests that this parity-odd coupling constant formulation cannot be generalized to the case of non-Abelian gauge coupling. This is because there appears no ratio of two scalar components which is group singlet to be identified with the coupling constant field if both of or one of the two vector multiplets are non-Abelian.

We have not discussed the other approach which Altendorfer et al ${ }^{2}$ pursued. As discussed by Falkowski $\mathbb{A}^{-}$and BKVP, anti-commute with the matrix $M^{i}{ }_{j}=\left(\sigma_{3}\right)^{i}{ }_{j}$ which is used in defining $Z_{2}$ parity of the fermions. The off-shell formulation for this approach is not difficult and actually was given by Zucker 177 for the case of linear multiplet compensator in the bulk. Half components of the bulk compensator yield a compensator multiplet on the brane. Both linear multiplet and hypermultiplet compensator in the bulk induces a chiral multiplet compensator on the brane which we denote by $\Sigma_{0}$. Generally in $4 \mathrm{D}$ superconformal framework, 18 the cosmological constant (without breaking supersymmetry) is supplied from the superpotential term $\left[\Sigma^{3}\right]_{F}$ of the chiral compensating multiplet $\Sigma$ with Weyl weight 1 provided that the $F$-component of $\Sigma$ develops a non-zero VEV. The brane tension term here, therefore, should be produced by giving a superpotential $F$ term $\left[\Sigma_{0}^{n}\right]_{F}$ on the brane. ( $n$ is a suitable power such that $n$ times Weyl weight of $\Sigma_{0}$ becomes 3.) But one can easily convince oneself that the $F$ component of this chiral compensator $\Sigma_{0}$ develops non-zero VEV if and only if the $U(1)_{R}$ generator $i \vec{q} \cdot \vec{\sigma}$ anti-commutes with $\sigma_{3}$. Moreover, this superpotential term can be multiplied 
by any function $g(S)$ of (Weyl weight 0 ) matter chiral multiplets $S_{i}$ living on the brane as $\left[\Sigma_{0}^{n} g(S)\right]_{F}$, and then it will clearly yield the brane tension of arbitrary magnitude which has no relation with the bulk cosmological term.

\section{Acknowledgements}

The authors would like to thank Hiroaki Nakano for his interest in this work. T. K. is supported in part by the Grants-in-Aid for Scientific Research No. 13640279 from Japan Society for the Promotion of Science and the Grants-in-Aid for Scientific Research on Priority Areas No. 12047214 from the Ministry of Education, Science, Sports and Culture, Japan.

\section{Appendix A \\ - Parametrization of hypermultiplet manifold -}

We concentrate on only the odd $\alpha$ components $\mathcal{A}_{i}^{\alpha=2 \hat{\alpha}-1}$ since the even $\alpha$ components $\mathcal{A}_{i}^{2 \hat{\alpha}}$ are given by the odd components by the reality as $\mathcal{A}^{2 \hat{\alpha}}{ }_{i}=\left(\mathcal{A}^{2 \hat{\alpha}-1}{ }_{j}\right)^{*} \epsilon_{j i}$. It was shown in I that the solution to the constraints $(5 \cdot 16)$ and $(5 \cdot 17)$ can be given in the form

$$
\mathcal{A}_{\text {odd }} \equiv \begin{aligned}
& 1 \\
& q
\end{aligned}\left(\begin{array}{cc}
\mathcal{A}^{a=1}{ }_{i=1} & \mathcal{A}^{a=1}{ }_{i=2} \\
\mathcal{A}^{a^{\prime}=3}{ }_{i=1} & \mathcal{A}^{a^{\prime}=3}{ }_{i=2} \\
\mathcal{A}^{\underline{\alpha}=\text { odd }_{i=1}} & \mathcal{A}^{\underline{\alpha}=\text { odd }}{ }_{i=2}
\end{array}\right)=U_{2, q}\left(\begin{array}{cc}
1 & 0 \\
0 & 1 \\
0 & 0
\end{array}\right)
$$

with a unitary matrix $U_{2, q} \in U(2, q)$, where $\mathcal{A}^{\underline{\alpha}=\text { odd }}{ }_{i}$ is a $q$-component complex vector for each $i=1,2$. This unitary matrix $U_{2, q}$ can be parametrized in the form 19)

$$
\begin{array}{rlrl}
U_{2, q} & =e^{\phi_{i} X^{i}} e^{\alpha^{i}\left(\phi, \phi^{\dagger}\right) X_{i}^{\dagger}} e^{\beta_{m}\left(\phi, \phi^{\dagger}\right) S_{m}}, \\
\phi_{i} X^{i} & \equiv\left(\begin{array}{ccc}
0 & 0 & 0 \\
0 & 0 & 0 \\
\phi_{1} & \phi_{2} & 0
\end{array}\right), & \alpha^{i} X_{i}^{\dagger} \equiv\left(\begin{array}{ccc}
0 & 0 & \alpha^{1} \\
0 & 0 & \alpha^{2} \\
0 & 0 & 0
\end{array}\right)
\end{array}
$$

by using two independent $q$-component vectors $\phi_{1}$ and $\phi_{2}$, where $S_{m}$ are the generators of 'unbroken' subgroup $H=U(2) \times U(q)$ and $\beta_{m}$ are complex generally. (Note that the broken generators $X^{i}, X_{i}^{\dagger}$ and their coefficients $\phi_{i}$ and $\alpha^{i}$ are all $q$-components for each $i=1,2$.) Actually the first factor $e^{\phi_{i} X^{i}}$ parametrizes the complex group coset $G^{C} / \hat{H}\left(G^{C}\right.$ : complex extension of $G, \hat{H}$ : complex subgroup whose generators are given by $\left\{X_{i}^{\dagger}, S_{m}\right\}$ ), and is just the basic variable in the non-linear realization theory by BKMU20 in supersymmetric theory. The coefficients $\alpha^{i}\left(\phi, \phi^{\dagger}\right)$ and $\beta_{m}\left(\phi, \phi^{\dagger}\right)$ are not independent parameters but are determined to be functions of $\phi_{i}$ and $\phi_{i}^{\dagger}$ by the demand that $U_{2, q}$ is a unitary matrix belonging to $U(2, q)$. 
It is not so easy to find explicit form of $\alpha^{i}\left(\phi, \phi^{\dagger}\right)$ and $\beta_{m}\left(\phi, \phi^{\dagger}\right)$, but we, fortunately here, can avoid the computation. Since $U_{2, q}$ is acting in Eq. (A-1) on the $(2+q) \times 2$ matrix whose last $q$ raws are all zero and the first two raws give $2 \times 2$ unit matrix, the third columns of the matrices $e^{\alpha^{i}\left(\phi, \phi^{\dagger}\right) X_{i}^{\dagger}}$ and $e^{\beta_{m}\left(\phi, \phi^{\dagger}\right) S_{m}}$ are irrelevant so that $e^{\alpha^{i}\left(\phi, \phi^{\dagger}\right) X_{i}^{\dagger}}$ can be replaced by 1 and $e^{\beta_{m}\left(\phi, \phi^{\dagger}\right) S_{m}}$ can be replaced by a $2 \times 2$ matrix $U^{C}$ acting from the right:

$$
\mathcal{A}_{\text {odd }}=U_{2, q}\left(\begin{array}{ll}
1 & 0 \\
0 & 1 \\
0 & 0
\end{array}\right)=e^{\phi_{i} X^{i}}\left(\begin{array}{ll}
1 & 0 \\
0 & 1 \\
0 & 0
\end{array}\right) U^{C}=\left(\begin{array}{c}
U^{C} \\
\left(\phi_{1} \phi_{2}\right) U^{C}
\end{array}\right)
$$

Now $U^{C}$ is determined by requiring that this form of $\mathcal{A}_{\text {odd }}$ satisfies the constraints (5.16) and $(5 \cdot 17)$, which is equivalent to the original condition that $U_{2, q}$ belongs to $U(2, q)$. Then, imposing the $S U(2)$ and $U(1)_{T}$ gauge conditions on $U^{C}$ to take the form

$$
U^{C}=\left(\begin{array}{cc}
a & 0 \\
\mathcal{A}^{3}{ }_{1} & b
\end{array}\right) \quad(a, b: \text { real, positive })
$$

we can find the solution of $U^{C}$ and hence of $\mathcal{A}_{\text {odd }}$ as given in Eq. (5.19) in the text.

The hypermultiplet scalar part of the Lagrangian is given as follows if written in the form before eliminating the auxiliary $S U(2)$ gauge field $V_{\mu}^{i j}$ :

$$
e^{-1} \mathcal{L}_{\mathrm{Hyp}}=+\mathcal{D}^{\mu} \mathcal{A}_{i}^{\bar{\alpha}} \mathcal{D}_{\mu} \mathcal{A}_{\alpha}^{i}=-\left|\mathcal{D}_{\mu} \mathcal{A}_{i}^{a}\right|^{2}-\left|\mathcal{D}_{\mu} \mathcal{A}_{i}^{a^{\prime}}\right|^{2}+\left|\mathcal{D}_{\mu} \mathcal{A}_{i}^{\underline{\alpha}}\right|^{2}
$$

where

$$
\begin{aligned}
& \mathcal{D}_{\mu} \mathcal{A}_{i}^{a}=\partial_{\mu} \mathcal{A}_{i}^{a}+\mathcal{A}_{j}^{a} V_{\mu}^{j}{ }_{i}-\left(W_{S \mu}+W_{T \mu}\right)\left(i \sigma_{3}\right)^{a}{ }_{b} \mathcal{A}_{i}^{b} \\
& \mathcal{D}_{\mu} \mathcal{A}_{i}^{a^{\prime}}=\partial_{\mu} \mathcal{A}_{i}^{a^{\prime}}+\mathcal{A}_{j}^{a^{\prime}} V_{\mu}^{j}{ }_{i}-W_{T \mu}\left(i \sigma_{3}\right)^{a^{\prime}}{ }_{{ }^{\prime}} \mathcal{A}_{i}^{b^{\prime}} \\
& \mathcal{D}_{\mu} \mathcal{A}_{i}^{\underline{\alpha}}=\partial_{\mu} \mathcal{A}_{i}^{\underline{\alpha}}+\mathcal{A}_{j}^{\underline{\alpha}} V_{\mu}^{j}{ }_{i}-W_{T \mu}\left(i \sigma_{3}\right)^{\underline{\alpha}}{ }_{\underline{\beta}} \mathcal{A}_{i}^{\underline{\beta}}-W_{\mu}^{I}\left(g t_{I}\right)^{\underline{\alpha}}{ }_{\underline{\beta}} \mathcal{A}_{i}^{\underline{\beta}} .
\end{aligned}
$$

If we neglect the $U(1)_{R}$ gauge interaction and other matter gauge interaction with generators $g t_{I}$ by setting $g_{R}=g=0$, then the Lagrangian (A.5) is simply rewritten in the following form by using $(2+q) \times 2$ complex matrix $\Phi=\mathcal{A}_{\text {odd }}$ :

$$
\begin{aligned}
& e^{-1} \mathcal{L}_{\mathrm{Hyp}}=2 \operatorname{tr}\left[\left(D^{\mu} \Phi\right)^{\dagger} \eta\left(D_{\mu} \Phi\right)\right], \quad D_{\mu} \Phi=\partial_{\mu} \Phi+\Phi A_{\mu}, \\
& \left(A_{\mu}\right)_{j}^{i}=\left(V_{\mu}\right)_{j}^{i}-i W_{T \mu} \delta_{j}^{i}, \quad \eta=\left(\begin{array}{ccc}
-1 & 0 & 0 \\
0 & -1 & 0 \\
0 & 0 & \mathbf{1}_{q}
\end{array}\right)
\end{aligned}
$$

$A_{\mu}$ now gives a $U(2)$ gauge field which comes from the combination of the $S U(2)$ and $U(1)_{T}$ symmetries. The four constraints (5·16) and (5.17) can be rewritten as

$$
\Phi^{\dagger} \eta \Phi=-\frac{1}{2} \mathbf{1}_{2}
$$


The Lagrangian $(\mathrm{A} \cdot 7)$ with constraints $\mathrm{A} \cdot \mathrm{B}$ ) clearly describes a nonlinear sigma model of Grassmannian manifold $U(2, q) / U(2) \times U(q)$.21) If the $U(2)$ auxiliary gauge field $A_{\mu}$ is eliminated the lagrangian is rewritten as

$$
e^{-1} \mathcal{L}_{\mathrm{Hyp}}=2 \operatorname{tr}\left[\left(\partial^{\mu} \Phi\right)^{\dagger} \eta\left(\partial_{\mu} \Phi\right)\right]+4 \operatorname{tr}\left[\left(\Phi^{\dagger} \eta \partial_{\mu} \Phi\right)^{2}\right]
$$

If the expression $(5 \cdot 19)$ of $\mathcal{A}_{\text {odd }}$ derived above is substituted for $\Phi$ here, then, it is easy to confirm that this Lagrangian can be written in the form

$$
\begin{aligned}
e^{-1} \mathcal{L}_{\text {Hyp }} & =\frac{\partial K(\phi, \bar{\phi})}{\partial \phi_{I} \partial \bar{\phi}_{\bar{J}}} \partial_{\mu} \phi_{I} \partial^{\mu} \bar{\phi}_{\bar{J}} \\
K(\phi, \bar{\phi}) & =-\ln \operatorname{det}\left[\left(\begin{array}{ccc}
1 & 0 & \phi_{1}^{\dagger} \\
0 & 1 & \phi_{2}^{\dagger}
\end{array}\right) \eta\left(\begin{array}{cc}
1 & 0 \\
0 & 1 \\
\phi_{1} & \phi_{2}
\end{array}\right)\right] \\
& =-\ln \left[\left(1-\left|\phi_{1}\right|^{2}\right)\left(1-\left|\phi_{2}\right|^{2}\right)-\left|\phi_{1}^{\dagger} \cdot \phi_{2}\right|^{2}\right]
\end{aligned}
$$

with $|\phi|^{2} \equiv \phi^{\dagger} \cdot \phi$. We see that this $K(\phi, \bar{\phi})$ is just the well-known Zumino-Aoyama form 22), 23) of the Kähler potential corresponding to the (Kählerian) Grassmannian manifold $U(2, q) / U(2) \times U(q)$, giving a special example of the general form presented by BKMU.20)

If the $U(1)_{R}$ gauge field is retained in the calculation, we see that the derivative $\partial_{\mu}$ in

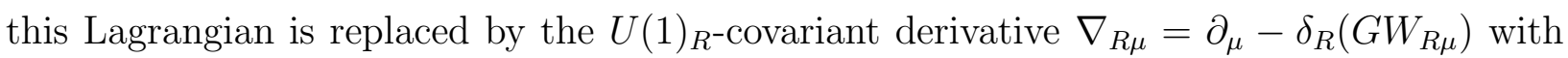
non-linear $U(1)_{R}$ transformation $\delta_{R}$.

\section{References}

[1] L. Randall and R. Sundrum, "An alternative to compactification," Phys. Rev. Lett. 83 (1999), 4690 hep-th/9906064.

[2] R. Altendorfer, J. Bagger and D. Nemeschansky, "Supersymmetric Randall-Sundrum Scenario", Phys. Rev. D63 (2001), 125025 hep-th/0003117

[3] T. Gherghetta and A. Pomarol, "Bulk Fields and Supersymmetry in a Slice of ADS", Nucl. Phys. B586 (2000), 141 hep-ph/0003129.

[4] A. Falkowski, Z. Lalak and S. Pokorski, "Supersymmetrizing Branes with Bulk in Five-Dimensional Supergravity", Phys. Lett. B491 (2000), 172 hep-th/0004093;

"Five-Dimensional Gauged Supergravities with Universal Hypermultiplet and Warped Brane Worlds", hep-th/0009167;

"Four-Dimensional Supergravities from Five-Dimensional Brane Worlds", hep-th/0102145.

[5] P. Hořava and E. Witten, Nucl. Phys. B460 (1996), 506, hep-th/9510209; Nucl. Phys. B475 (1996), 94, hep-th/9603142. 
[6] A. Lukas, B. A. Ovrut, K.S. Stelle and D. Waldram, Phys. Rev. D59 (1999), 086001 hep-th/9803235; Nucl. Phys. B552 (1999), 246 hep-th/9806051.

[7] E. Bergshoeff, R. Kallosh and A. Van Proeyen, "Supersymmetry in Singlar Spaces," JHEP 0010 (2000) 033, hep-th/0007044.

[8] M. Günaydin, G. Sierra and P.K. Townsend, Nucl. Phys. B242 (1984), 244; Nucl. Phys. B253 (1985), 573.

[9] T. Kugo and K. Ohashi, "Off-Shell $d=5$ Supergravity Coupled to a Matter-YangMills System", Prog. Theor. Phys. 105 (2001), 323 hep-ph/0010288.

[10] T. Fujita and K. Ohashi, "Superconformal Tensor Calculus in Five Dimensions", hep-th/0104130.

[11] T. Kugo, K. Ohashi, "Supergravity Tensor Calculus in 5D from 6D," Prog.Theor.Phys. 104 (2000) 835 hep-ph/0006231.

[12] E. Bergshoeff, S.Cucu, M. Derix, T. de Wit, R. Halbersma and A. Van Proeyen, "Weyl multiplets of $N=2$ Conformal Supergravity in Five Dimensions," hep-th/0104113.

[13] B. de Wit, P. G. Lauwers and A. Van Proeyen, Nucl. Phys. B255 (1985), 569.

[14] E. Bergshoeff, E. Sezgin and A. Van Proeyen, Nucl. Phys. B264 (1986), 653.

[15] S. Cecotti, S. Ferrara and L. Girardello, Phys. Lett. B213 (1988), 443; Int. J. Mod. Phys. A4 (1989), 2475.

[16] R. Britto-Pacumio, A. Strominger and A. Volovich, JHEP 9911 (1999), 013 hep-th/9905211.

K. Behrndt and M. Cvetič, "Gauging of $N=2$ Supergravity Hypermultiplet and Novel Renormalization Group Flows", hep-th/0101007.

[17] M. Zucker, "Supersymmetric brane worlds scenarios from off-shell supergravity," hep-th/0009083.

[18] T. Kugo and S. Uehara, Nucl. Phys. B226 (1983), 49; Prog. Theor. Phys. 73 (1985), 235.

[19] K. Itoh, T. Kugo and H. Kunitomo, Nucl. Phys. B263 (1986), 295; Prog. Theor. Phys. 75 (1986), 386.

[20] M. Bando, T. Kuramoto, T. Maskawa and S. Uehara, Phys. Lett. 138B (1984), 94;

Prog. Theor. Phys. 72 (1984), 313; 72 (1984), 1207.

[21] M. Bando, T. Kugo and K. Yamawaki, Phys. Reports 164 (1988), 217.

[22] B. Zumino, Phys. Lett. 87B (1979), 293.

[23] S. Aoyama, Nouvo Cim. A57 (1980), 176. 